\title{
Development of a protocol for the auto-generation of explicit aqueous-phase oxidation schemes of organic compounds
}

\author{
Peter Bräuer ${ }^{1, \mathrm{a}}$, Camille Mouchel-Vallon ${ }^{2, \mathrm{~b}}$, Andreas Tilgner ${ }^{1}$, Anke Mutzel $^{1}$, Olaf Böge $^{1}$, Maria Rodigast $^{1, \mathrm{c}}$, \\ Laurent Poulain $^{1}$, Dominik van Pinxteren ${ }^{1}$, Ralf Wolke ${ }^{3}$, Bernard Aumont ${ }^{2}$, and Hartmut Herrmann ${ }^{1}$ \\ ${ }^{1}$ Atmospheric Chemistry Department (ACD), Leibniz Institute for Tropospheric Research (TROPOS), Permoserstr. 15, \\ 04318 Leipzig, Germany \\ ${ }^{2}$ Laboratoire Interuniversitaire des Systèmes Atmosphériques, UMR CNRS/INSU 7583, \\ Université Paris Est Créteil et Université Paris Diderot, Institut Pierre Simon Laplace, 94010, Créteil, France \\ ${ }^{3}$ Modelling of Atmospheric Processes Department (MAPD), Leibniz Institute for Tropospheric Research (TROPOS), \\ Permoserstr. 15, 04318 Leipzig, Germany \\ a now at: Wolfson Atmospheric Chemistry Laboratories and National Centre for Atmospheric Science, University of York, \\ York, YO10 5DD, UK \\ ${ }^{b}$ now at: National Center for Atmospheric Research, Boulder, Colorado, USA \\ ${ }^{c}$ now at: Indulor Chemie GmbH \& Co. KG Produktionsgesellschaft Bitterfeld, 06749 Bitterfeld-Wolfen, Germany
}

Correspondence: Hartmut Herrmann (herrmann@tropos.de)

Received: 19 December 2018 - Discussion started: 16 January 2019

Revised: 17 May 2019 - Accepted: 27 May 2019 - Published: 19 July 2019

\begin{abstract}
This paper presents a new CAPRAM-GECKOA protocol for mechanism auto-generation of aqueous-phase organic processes. For the development, kinetic data in the literature were reviewed and a database with 464 aqueousphase reactions of the hydroxyl radical with organic compounds and 130 nitrate radical reactions with organic compounds has been compiled and evaluated. Five different methods to predict aqueous-phase rate constants have been evaluated with the help of the kinetics database: gas-aqueous phase correlations, homologous series of various compound classes, radical reactivity comparisons, Evans-Polanyi-type correlations, and structure-activity relationships (SARs). The quality of these prediction methods was tested as well as their suitability for automated mechanism construction. Based on this evaluation, SARs form the basis of the new CAPRAM-GECKO-A protocol. Evans-Polanyi-type correlations have been advanced to consider all available $\mathrm{H}$ atoms in a molecule besides the $\mathrm{H}$ atoms with only the weakest bond dissociation enthalpies (BDEs). The improved EvansPolanyi-type correlations are used to predict rate constants for aqueous-phase $\mathrm{NO}_{3}$ and organic compounds reactions.

Extensive tests have been performed on essential parameters and on highly uncertain parameters with limited ex-
\end{abstract}

perimental data. These sensitivity studies led to further improvements in the new CAPRAM-GECKO-A protocol but also showed current limitations. Biggest uncertainties were observed in uptake processes and the estimation of Henry's law coefficients as well as radical chemistry, in particular the degradation of alkoxy radicals. Previous estimation methods showed several deficits, which impacted particle growth.

For further evaluation, a 1,3,5-trimethylbenzene oxidation experiment has been performed in the aerosol chamber "Leipziger Aerosolkammer" (LEAK) at high relative humidity conditions and compared to a multiphase mechanism using the Master Chemical Mechanism (MCMv3.2) in the gas phase and using a methylglyoxal oxidation scheme of about 600 reactions generated with the new CAPRAM-GECKO-A protocol in the aqueous phase. While it was difficult to evaluate single particle constituents due to concentrations close to the detection limits of the instruments applied, the model studies showed the importance of aqueous-phase chemistry in respect to secondary organic aerosol (SOA) formation and particle growth. The new protocol forms the basis for further CAPRAM mechanism development towards a new version 4.0. Moreover, it can be used as a supplementary tool for aerosol chambers to design and analyse experiments 
of chemical complexity and help to understand them on a molecular level.

\section{Introduction}

The ubiquitous abundance of organic compounds in natural and anthropogenically influenced ecosystems impacts climate, air quality, human health, the oxidation capacity of the troposphere, crop yields, particle growth, and composition. Their detrimental effects cause enormous economic cost (Boucher et al., 2013; Brasseur et al., 2003; Dunmore et al., 2015; Hallquist et al., 2009; Pereira et al., 2018). With large annual emissions of about $1300 \mathrm{Tg} \mathrm{C} \mathrm{a}^{-1}$ (Goldstein and Galbally, 2007), non-methane volatile organic compounds have been a research interest for many decades. Despite intense research efforts, the system of organic compounds is still insufficiently understood because of its complexity. The large emissions lead to a large mass of organic compounds in the atmosphere but also to a large variety of organic compounds. Currently, $10^{4}$ to $10^{5}$ different organic compounds have been identified but are assumed to be only a small fraction of the actual number (Goldstein and Galbally, 2007).

The multiphase nature of the oxidation of organic compounds further contributes to the complexity. Organic compounds are ubiquitously found in aerosol particles around the globe with contributions between $20 \%$ and $90 \%$ of the total aerosol mass (Jimenez et al., 2009). While primary organic aerosol (POA) is an additional source of organic compounds in the atmosphere to direct gas-phase emissions, gasto-particle conversion, multiphase, and heterogeneous processes form secondary organic aerosol (SOA) influence the composition in either phase (Ervens et al., 2011; Hallquist et al., 2009; Jimenez et al., 2009). With aerosol water being in excess of dry aerosol mass by a factor of 2 to 3 (Ervens et al., 2011), aqueous-phase chemistry plays an important role in the degradation of organic compounds. Organic aerosol oxidation state, size, and product distribution as well as relative humidity dependence can only be explained by aqueousphase chemistry forming secondary organic aerosol (aqSOA; Ervens et al., 2011).

Numerical modelling provides a useful tool for the analysis of such comprehensive and complex processes. Comprehensive benchmark mechanisms exist for either the gas phase (e.g. the Master Chemical Mechanism, MCM, http: $/ / \mathrm{mcm}$.leeds.ac.uk/, last access: 5 October 2015; Jenkin et al., 2003; Saunders et al., 2003) or the aqueous phase (e.g. the Chemical Aqueous Phase Radical Mechanism, CAPRAM, http://projects.tropos.de/capram/, last access: 3 December; Herrmann et al., 2005, with latest updates by Bräuer et al., 2013). With a growing mechanism size in either phase of currently about 17000 gas-phase (MCM v3.3.1) and 777 aqueous-phase reactions (CAPRAM 3.0), mechanism auto-generation becomes increasingly important (see also
Vereecken et al., 2018). This method has several advantages over manual mechanism construction. Among its key strong points are a reduced proneness to errors as any errors embedded in the generation routines produce systematic errors in the output chemical mechanisms, which are easier to detect than random errors in manual mechanisms. Most importantly, with suitable estimation methods for the prediction of the necessary kinetic and mechanistic data, it is possible to overcome the inevitable lack of experimental data for the large number of organic compounds measured in field and laboratory experiments or produced by mechanism autogeneration. The detailed explicit mechanism produced with an auto-generation method can help to interpret experimental data (e.g. La et al., 2016; Lee-Taylor et al., 2015; McVay et al., 2016). Modelling can suggest isomer information along with detailed information about the production and degradation processes.

Currently, atmospheric organic chemistry is more widely studied and understood in the gas phase than in the aqueous phase, whether through experimental or modelling studies (Ervens, 2015). The current study aims to reduce this gap by providing a protocol for the auto-generation of comprehensive aqueous-phase mechanisms. While with the Generator for Explicit Chemistry and Kinetics of Organics in the Atmosphere (GECKO-A; Aumont et al., 2005), tools for mechanism auto-generation are already available for the gas phase, only first attempts have been presented for the aqueous phase with the study by Li and Crittenden (2009). MouchelVallon et al. (2013) investigated a protocol for phase transfer processes between the gas and the aqueous phase and effects on the composition in either phase. The current study takes the study by Mouchel-Vallon et al. (2013) a step further with a new protocol for mechanism auto-generation of chemical processes in the aqueous phase. A new protocol has been developed and incorporated in the expert system GECKO-A. Test mechanisms were generated and validated against experiments performed at the aerosol chamber LEAK (Leipziger Aerosolkammer; Mutzel et al., 2016). Beforehand, an extensive literature review of kinetic data and suitable estimation methods for the prediction of kinetic and mechanistic data for aqueous-phase reactions of organic compounds was performed to ensure the generation of comprehensive, state-of-the-art aqueous-phase mechanisms.

\section{Evaluation of kinetic data and prediction methods}

\subsection{Evaluation of experimental kinetic data}

For the construction of a protocol for mechanism autogeneration, a large database with kinetic data is a prerequisite. Data are used directly to assign rate constants to reactions created by the mechanism generator and indirectly to evaluate or advance current prediction methods for missing rate constants based on the known experimental values. The 
present study focuses on aliphatic organic compounds; however, the kinetics database includes aromatic compounds of atmospheric interest for completeness. Overall, 464 aqueousphase hydroxyl radical $(\mathrm{OH})$ and 130 nitrate radical $\left(\mathrm{NO}_{3}\right)$ reactions with organic compounds have been reviewed and can be found in section S1 of the Supplement (ESM). The database includes compound classes of atmospheric interest, i.e. alkanes, alcohols (including di- and polyols), carbonyl compounds, carboxylic acids and diacids, esters, ethers, unsaturated, cyclic, aromatic compounds, and polyfunctional compounds composed of these functionalities.

The kinetics database is based on existing recommendations and compilations (Buxton et al., 1988; Ross et al., 1998; Herrmann, 2003; Herrmann et al., 2010) with additional data collected from recent literature. In exceptional cases, where no other data existed, data from unpublished TROPOS measurements have been used for the database as well. In cases, where no clear preference to a dataset could be given, averaging was used.

\subsection{Simple correlations}

Besides the review of kinetic data, it is equally important to review estimation methods for the prediction of missing kinetic data in auto-generated mechanisms. A number of prediction methods exist, which include straightforward correlations that are easy to implement in automated tools for mechanism generation. These correlations include gas-aqueousphase correlations, extrapolations of homologous series of compound classes, and reactivity comparisons between different radical oxidants.

These methods were therefore evaluated for their use in mechanism generators. Correlations were derived for the various compound classes, and absolute errors are analysed with box plots. Results from the analysis are shown in Fig. 1, while a detailed analysis is given in Sect. S2 of the ESM. For such simplistic approaches, good results are obtained from these methods, and one can expect to at least predict the correct order of magnitude for a given rate constant (see Fig. 1). In general, slightly negative absolute errors are observed in Fig. 1; thus the predictions tend to overpredict the rate constants somewhat. The largest deviations of the absolute errors of the logarithmized rate constants range from -2.1 to 0.9 . Simple structures like pure hydrocarbons are predicted far more accurately than more complex structures. The largest deviations are observed for carbonyls and carboxylic acids. Moreover, often the smallest members of a compound class show large deviations from the general trend as in the case of carboxylic acids. For alkanes, methane is excluded in the evaluation of homologous series in Fig. 1 as its absolute log error of 16.8 does not fit in the scale of the plot.

While the easy implementation of the above correlations in computer-assisted tools make their usage desirable, they are unsuitable for mechanism auto-generation for several reasons. Overall, their accuracy is satisfactory but can be im-

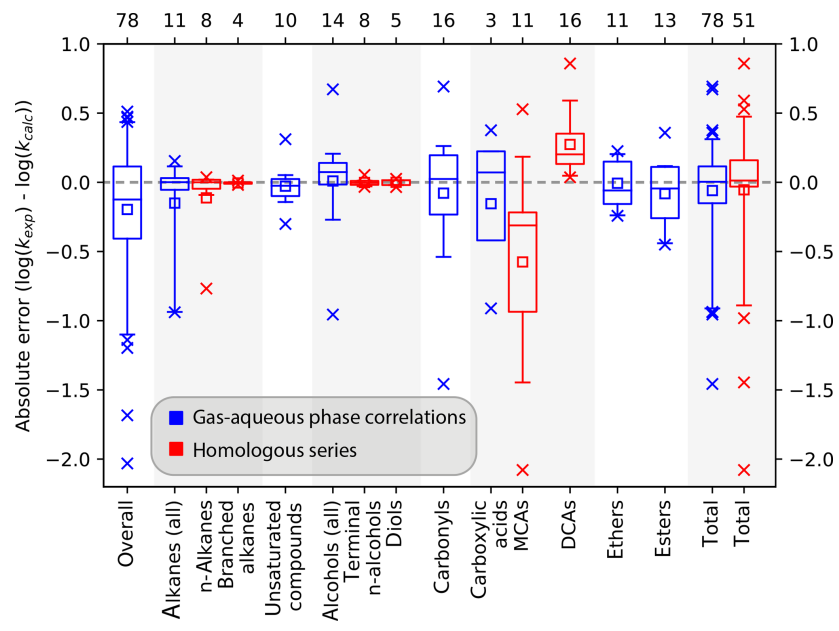

Figure 1. Box plots of the absolute errors of the logarithmized experimental versus predicted $\mathrm{OH}$ rate constants for the prediction of $\mathrm{OH}$ rate constants with gas-aqueous-phase correlations (blue boxes) and extrapolations of homologous series (red boxes). Boxes represent the quartiles of the absolute errors, whiskers the $95 \%$ confidence interval, data points outside this are shown explicitly. The arithmetic mean is represented by a square. The "overall" box represents absolute errors from a gas-aqueous-phase correlation derived from a fit of all data, while the total column denotes the evaluation of all errors compiled from the analysis of the various compound classes.

proved. More importantly, all of the above correlations have a very restricted applicability. For gas-aqueous-phase correlations, gas-phase kinetic data are needed. For complex systems such as CAPRAM-GECKO-A, many data had to be estimated, which would increase errors to unsatisfactory levels. For radical comparisons, the lack of the needed aqueousphase data is even worse. The prediction with homologous series is restricted to very distinct molecule structures as detailed in the ESM. Therefore, the focus of this study is on more complex correlations and prediction methods.

\subsection{Evans-Polanyi-type correlations}

More advanced correlations for the prediction of rate constants of organic compounds are Evans-Polanyi correlations. Originally developed for the gas phase by Evans (1938), they have been successfully applied to the aqueous phase (see, e.g. Herrmann and Zellner, 1998; Hoffmann et al., 2009). In an Evans-Polanyi-type correlation, a linear relationship between the activation energy and the bond dissociation enthalpy (BDE) of a molecule is derived by the following:

$E_{\mathrm{A}}=a^{\prime}+b^{\prime} \cdot \operatorname{BDE}(\mathrm{CH})$.

Using the Arrhenius expression in its logarithmized form $\ln$ $k=\ln (A)-E_{\mathrm{A}} / \mathrm{RT}$ and assuming a rupture of only the weak- 
est $\mathrm{C}-\mathrm{H}$ bonds in molecule leads to the following:

$\log \left(k_{\mathrm{H}}\right)=\log \left(\frac{k_{2 \mathrm{nd}}}{n_{\mathrm{H}}}\right)=a-b \cdot \mathrm{BDE}(\mathrm{CH})$,

where $a=\log \left(A / n_{\mathrm{H}}\right)-a^{\prime} /(\mathrm{RT} \ln 10)$ and $b=b^{\prime} /(\mathrm{RT} \ln 10)$, $n_{\mathrm{h}}$ is the number of $\mathrm{H}$ atoms bonded with the lowest BDE in a molecule, $R$ is the universal gas constant, and $T$ is the temperature. $A$ is the pre-exponential factor of the Arrhenius equation, which has to be similar for all reactants correlated to fulfil Eq. (2). $k_{\mathrm{H}}$ is the second-order rate constant of the reaction scaled by the number of weakest $\mathrm{C}-\mathrm{H}$ bonds. The method relies on BDEs as input for the correlation, which can be estimated reliably with a precision of $\pm 8 \mathrm{~kJ} \mathrm{~mol}^{-1}$ (Benson, 1976). The method by Benson (1976) estimates the strength of a bond as a function of the adjacent atoms/molecular groups.

\subsubsection{OH rate constant prediction}

The kinetics database was used to derive Evans-Polanyi-type correlations, which are plotted in Fig. S5 in the ESM. Parameters for the regression equations and further statistical data are given in Table S8 in the ESM. Even for a large database as used in this assessment, a reliable correlation is hardly achieved (see Fig. S5 in the ESM). Several outliers can be found in the dataset. A critical evaluation of the data is necessary and depending on this evaluation, several possible correlations can be found. Furthermore, there are several cases where data points have exactly the same BDE but vary in their reaction rate constants by more than 1 order of magnitude. The reason for this behaviour is the correlation of the rate constants to only the weakest $\mathrm{BDE}$, which corresponds to the main radical attack site in a molecule or the main reaction channel. When correlating a homologous series of compounds to the bond dissociation enthalpy, the lowest BDE in each molecule varies only slightly. On the other hand, rate constants increase significantly with the carbon number due to side attacks of the radical oxidants at the remaining carbon skeleton. With increasing chain length, side attacks become more likely even if the BDE is higher compared to the weakest bond. With the correlation of $\log \left(k_{\mathrm{H}}\right)$ against only the smallest BDE of a molecule, this fact cannot be considered in the correlation.

Accordingly, the use of Evans-Polanyi correlations in this way results in large uncertainties. The evaluation of the absolute errors of the predicted versus experimental data with box plots confirms the discussion above. Relatively large errors are seen for carbonyl compounds and carboxylic acids, while errors are somewhat smaller for alcohol compounds and alkanes (see Fig. 2). The performance is not significantly better than the performance of the much simpler correlations described in the preceding section. However, a major advantage of Evans-Polanyi-type correlations is the much wider applicability.

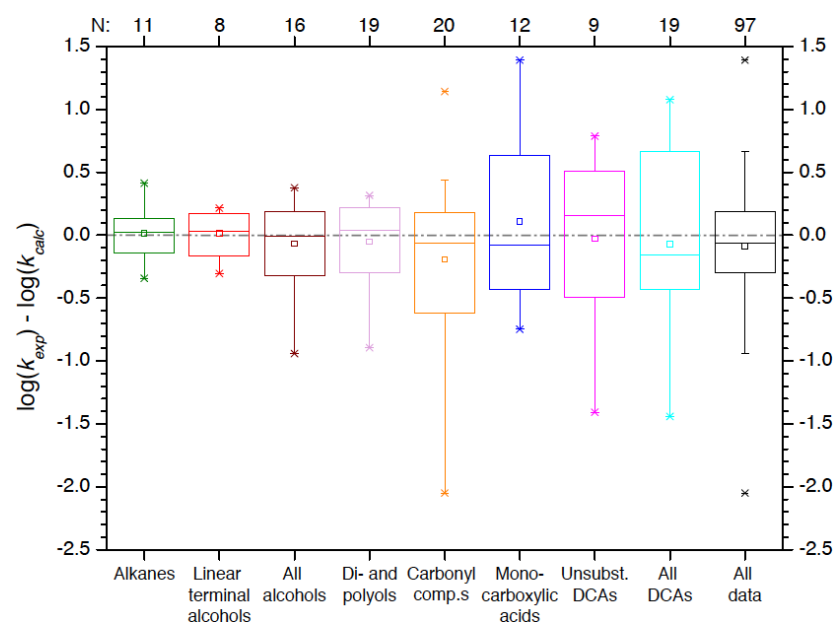

Figure 2. Box plots of the absolute errors of Evans-Polanyi-type correlations of hydroxyl radical reactions with organic compounds distinguished by compound class.

For further details, the prediction method has been reanalysed with the help of scatter plots, where the predicted data derived from the correlations are plotted over the experimental data. In a perfect correlation, all data should line up on the $1: 1$ line in such a plot with a coefficient of determination $R^{2}$ of 1 . Any deviations of the $y$ intercept of the regression line from 0 or of the slope from 1 indicate a general bias of the prediction method. Reductions of the coefficient of determination are due to deviations of the single predictions.

Figure 3 shows that, except for the pure hydrocarbons, rate constants of fast reacting compounds are underpredicted while rate constants of smaller compounds are overpredicted. This fact demonstrates the limitations of the current EvansPolanyi-type correlations to accurately predict rate constants of larger compounds as it was already seen during the derivation of the correlation. Faster rate constants in the dataset belong to larger compounds. Hence, the increase of their observed rate constants is larger than the increase expected from their lowest BDE due to increased side attacks of the hydroxyl radical at the remaining carbon skeleton. As these side attacks are not considered in the correlation, where only the weakest bound $\mathrm{H}$ atoms of the major reaction pathway are correlated against $\log \left(k_{\mathrm{H}}\right)$, an underprediction is inevitable. As a consequence, the regression line of the correlation is tilted resulting in an overprediction of smaller or slow-reacting compounds.

\subsection{2 $\mathrm{NO}_{3}$ rate constant prediction}

The much smaller dataset of available $\mathrm{NO}_{3}$ reactions of aliphatic compounds further reduces the accuracy of EvansPolanyi-type correlations. It is considerably harder to derive reliable correlations, which can be achieved only for alcohol compounds and carboxylic acid with an appreciable coeffi- 


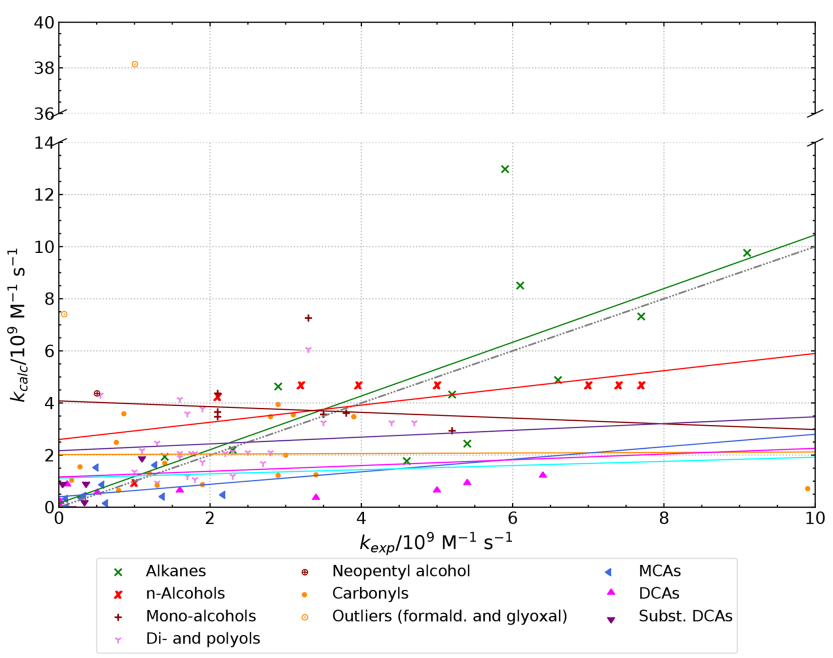

Figure 3. Plot of predicted versus experimental data for EvansPolanyi correlations of $\mathrm{OH}$ radical reactions with organic compounds differentiated by compound class. Parameters of the regression lines are given in Table S8 in the ESM. The regression lines have the same colour code as the data points. The black dasheddotted line is the line of same reactivity.

cient of determination $R^{2}$ between 0.6 and 0.7. Correlations are shown in Fig. 4a with the respective statistical data given in Table S9 in the ESM.

Predictions of $\mathrm{NO}_{3}$ reactions with organic compounds inherit large errors. For a large fraction, the right order of magnitude is not even predicted. Again, an underprediction of fast reactions (or large molecules) is observed, while the rate constants of smaller or slow-reacting compounds are overpredicted (see Fig. 4a) as discussed for $\mathrm{OH}$ in the previous subsection.

\subsubsection{Advanced Evans-Polanyi-type correlations}

The evaluation of current Evans-Polanyi-type correlations has shown strong limitations for the use of automated prediction methods, which aim at large molecules where the formation of many intermediate compounds makes manual mechanism construction infeasible. As the accuracy of the predictions of rate constants for these compounds is significantly reduced, a revision and improvement of the current correlations is needed and is described in this subsection.

\section{Development of improved Evans-Polanyi-type correlations}

The need to improve Evans-Polanyi-type correlations for larger compounds, where minor reaction channels become increasingly important as outlined in the preceding subsections, led to a revised correlation method where the sum of all BDEs of bonds including hydrogen atoms $\left(\sum \mathrm{BDE}\right)$ was correlated against the overall second-order rate constant.

$\log \left(k_{\exp }\right)=a-b \cdot \sum \mathrm{BDE}$

The consideration of minor pathways is achieved by including all BDEs rather than only the weakest ones and the overall second-order rate constant instead of the second-order rate constants scaled to the number of weakest $\mathrm{H}$ atoms. A certain disadvantage of this method is that information about branching ratios and reaction products cannot be provided.

\section{OH radical rate constant predictions}

Compared to previous Evans-Polanyi-type correlations, significant changes are observed as can be seen from Fig. 5. The range of $\sum \mathrm{BDE}$ is much broader ranging from about 500 to $8000 \mathrm{~kJ} \mathrm{~mol}^{-1}$ compared to a BDE range of about 360 to $440 \mathrm{~kJ} \mathrm{~mol}^{-1}$. More importantly, in the new correlation there is a positive correlation between $\sum \mathrm{BDE}$ and $k_{\text {exp }}$. The positive correlation derives from the fact that $\sum \mathrm{BDE}$ is correlated to all abstractable $\mathrm{H}$ atoms and that $k_{\text {exp }}$ increases with larger molecules, i.e. an increasing number of $\mathrm{H}$ atoms or $\sum$ BDE. In the original Evans-Polanyi-type correlations, a negative correlation is observed due to higher reactivities at molecular sites with low BDEs. The most striking differences are quadratic correlations in the advanced EvansPolanyi-type correlations compared to linear correlations previously. In the new correlations, rate constants plotted over $\sum \mathrm{BDE}$ follow downward-opened parabolas. All data fit with in an upper and lower parabola, which converge towards $\log \left(k_{\exp } / \mathrm{M}^{-1} \mathrm{~s}^{-1}\right)=10$ and $\sum \mathrm{BDE}=8000 \mathrm{~kJ} \mathrm{~mol}^{-1}$ in the $\log \left(k_{\text {exp }}\right)$ over $\sum \mathrm{BDE}$ diagram.

A likely reason for the different behaviour of the old and the new regressions is the diffusion limit of reactions. The diffusion limit does not come into effect in the old correlation, where the partial rate constant $\left(k_{\mathrm{H}}\right)$ refers to only the weakest bound $\mathrm{H}$ atoms. When the overall rate constant is considered, the diffusion limit is reached for high values of $\sum B D E$. Thus, despite an increasing $\sum B D E$ due to an increasing number of abstractable $\mathrm{H}$ atoms, the rate constants might not increase accordingly as they are levelling off and undergo a transition to control by diffusion rather than by chemical control. This view is supported by the upper limit of all data around $\log \left(k_{\exp } / \mathrm{M}^{-1} \mathrm{~s}^{-1}\right)=10$, the approximate diffusion limit of rate constants of $\mathrm{OH}$ with organic compounds (e.g. Haag and Yao, 1992; Schöne et al., 2014).

The correlations show significant improvements with coefficients of determination $R^{2}$ up to 0.99 (see Fig. S6 and Table S10 in the ESM). A weak correlation is still observed for ketones $\left(R^{2}=0.24\right)$ and alcohol compounds except linear terminal alcohols $\left(R^{2}=0.35\right)$. Monocarboxylic acids show only a moderate correlation $\left(R^{2}=0.55\right)$. The better performance can also be evaluated from the absolute errors analysed as box plots in Fig. 6. Almost all data are predicted with the correct order of magnitude. The weak correlations of the above-mentioned compound classes are reflected by 

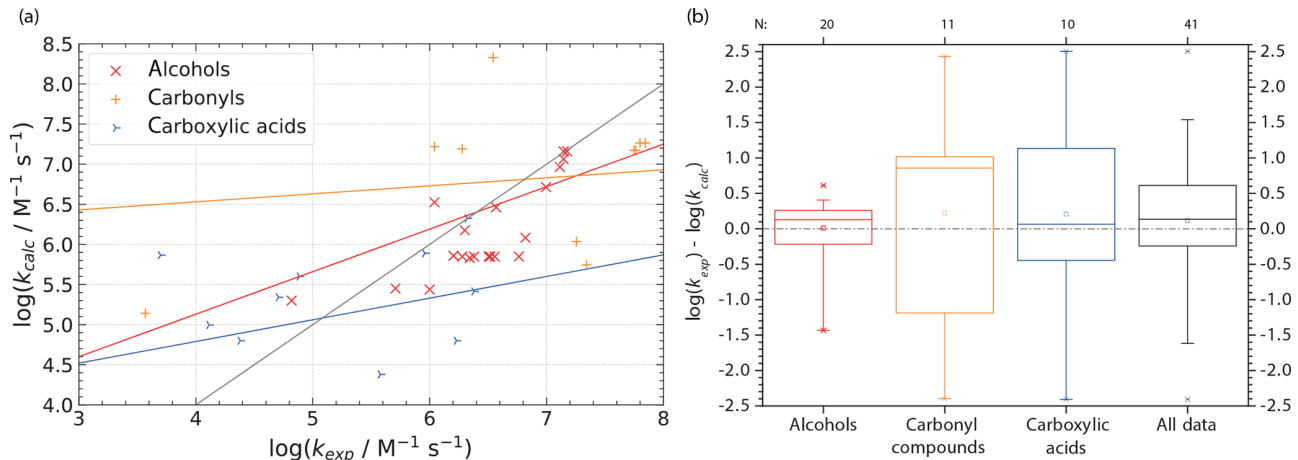

Figure 4. Evaluation of predicted versus experimental data for Evans-Polanyi-type correlations of $\mathrm{NO}_{3}$ radical reactions with organic compounds using scatter plots (a) and analysis of absolute errors (b) distinguished by compound class. Parameters for the linear regression lines are given in Table S9 in the ESM.

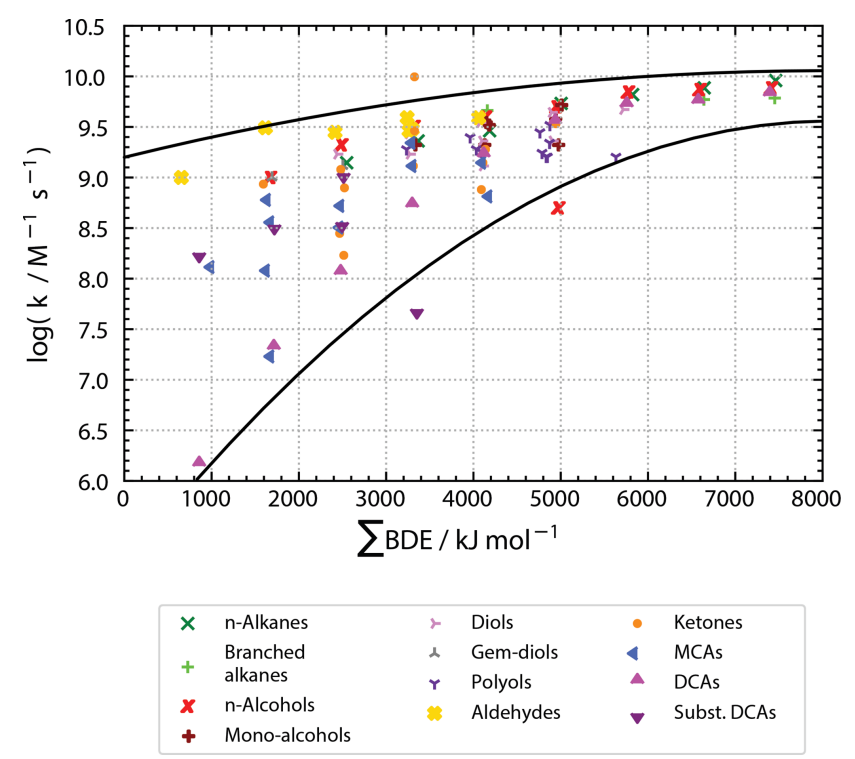

Figure 5. Plot of experimentally determined second-order rate constants versus accumulated bond strengths of all bonds containing hydrogen atoms in a molecule ( $\Sigma \mathrm{BDE}$ ) distinguished by compound classes. Black lines mark upper and lower boundary parabolas with most of the data lying within these boundaries.

larger errors. More importantly, the objective to reduce biases of larger molecules was achieved as can be observed from the scatter plot of the calculated over experimental data in Figure S7 in the ESM. Except for ketones, regression lines now have a slope close to 1 (between 0.76 and 1.16). Omitting the outlier acetylacetone from the analysis of ketones gives a slope of the regression line of 0.54 , which is still a big improvement compared to the original correlation. Although an omission of data points for the derivation of a correlation is not ideal, a large modified $Z$ score after Iglewicz and Hoaglin (1993) (see Eq. 4) of 4.21 justifies the removal of the data point from the dataset. Acetylacetone is the only compound which has a modified $Z$ score well above the sug-

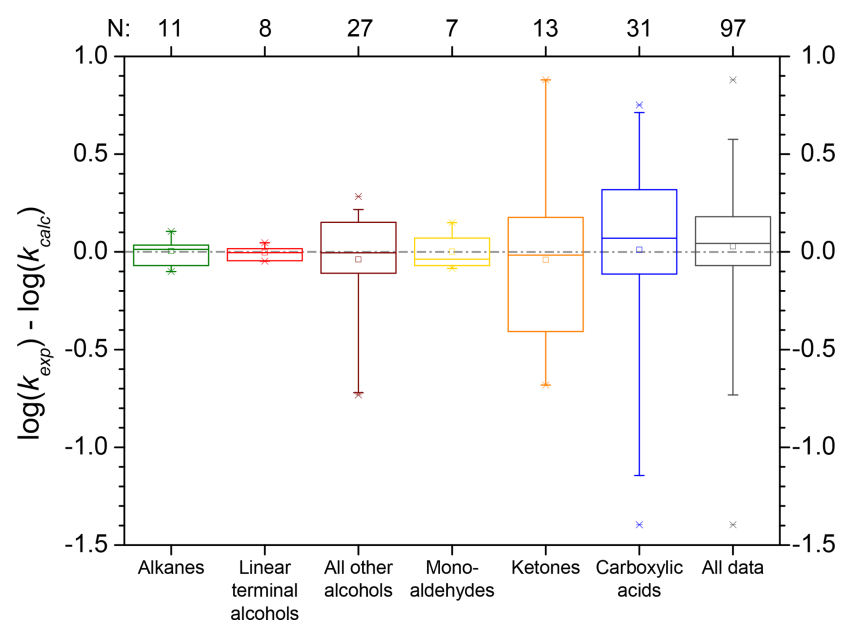

Figure 6. Box plots of absolute errors of second-order OH rate constants predicted with the advanced Evans-Polanyi-type correlations versus experimental data for the various compound classes as well as all data.

gested threshold of 3.5 (Iglewicz and Hoaglin, 1993).

$Z_{i}=\frac{0.6745\left(x_{\mathrm{i}}-\tilde{x}\right)}{\operatorname{MAD}}$

In Eq. (4), $x_{\mathrm{i}}$ is the individual measurement, $\tilde{x}$ the median, and MAD the median absolute deviation.

\section{$\mathrm{NO}_{3}$ radical rate constant predictions}

When deriving the advanced Evans-Polanyi-type correlations for nitrate radical reactions with organic compounds, a major difference to hydroxyl radical reactions is observed. $\mathrm{NO}_{3}$ reactions follow a linear trend rather than a quadratic correlation. The additional quadratic polynomial does not reduce errors significantly. An explanation for the different behaviour can be found again in the diffusion limit of both reactions. The diffusion limit of nitrate radical reactions is only insignificantly smaller compared to hydroxyl radical re- 
actions $\left(\sim 9.5 \times 10^{9} \mathrm{M}^{-1} \mathrm{~s}^{-1}\right.$ vs. $\left.\sim 10^{10} \mathrm{M}^{-1} \mathrm{~s}^{-1}\right)$. However, rate constants for nitrate radical reactions are of the order of $10^{4}$ to $10^{8} \mathrm{M}^{-1} \mathrm{~s}^{-1}$. Most reactions are below $1 \%$ of their diffusion-controlled limit. Therefore, the diffusion limit does not affect the correlation. Even though a quadratic correlation could be applied, values are so far away from the maximum of the parabola that a linear equation is an equally valid approximation.

The correlations are shown in Fig. 7 with the raw data in panel (a) and the final correlations in panel (b). The respective statistical data are provided in Table S11 and S12 in the ESM. Both linear (solid lines) and quadratic regressions (dashed lines) are shown in Fig. 7b. Another advantage of the advanced Evans-Polanyi-type correlations is that certain compound classes can be grouped together without a loss of accuracy. This increases the number of data points used for each regression and, therefore, its reliability. For the derivation of the final correlations, data from tert-butanol and acetone have not been considered due to their high modified $Z$ scores of 5.55 and 4.57 respectively, which are well above the suggested threshold of 3.5 (Iglewicz and Hoaglin, 1993).

As can be seen from Fig. 7b and the statistical data in Tables S11 and S12 in the ESM, only a weak correlation with large associated errors is observed for carboxylic acids. Moreover, for this compound class, a negative correlation is observed for which the reasons remain unclear. More data are needed to constrain a more reliable correlation for these types of molecules and their respective $\mathrm{NO}_{3}$ reactions.

Overall, the advanced Evans-Polanyi-type correlations perform well with significantly reduced errors as can be seen from the box plot analysis in Fig. S8 in the ESM. The correct order of magnitude is met for almost all compounds and usually errors are much smaller. Only one carboxylic and two dicarboxylic acids are not predicted within the correct order of magnitude. Biases of the slopes of the regression lines in the scatter plot of the calculated over experimental data are significantly reduced except for the very uncertain correlation of carboxylic acids (see Fig. S7 in the ESM).

\subsection{Structure-activity relationships}

A more sophisticated prediction of rate constants of the hydroxyl radical with organic compounds can be achieved by means of structure-activity relationships (SARs). In a SAR, the assumption is made that a molecule can be split into increments. Rate constants are assigned to the individual increments, which are modulated by the effects of the neighbouring groups in the $\alpha$ position and, in the case of the SAR by Doussin and Monod (2013), in $\beta$ position as well as ring effects of the molecule. The overall reaction rate is the sum of the incremental rate constants. Two structure-activity relationships from the literature have been compared - the SAR by Minakata et al. (2009) and the one by Monod and Doussin (2008) with updates by Doussin and Monod (2013).

\subsubsection{SAR by Minakata et al. (2009)}

A big advantage of the SAR of Minakata et al. (2009) is the large dataset of 434 aqueous-phase reaction rate constants covering all important compound classes for tropospheric aqueous-phase chemistry, including alkanes, alcohols, carbonyl compounds, carboxylic acids, ethers, esters, sulfur-, nitrogen- and phosphorus-containing species, polyfunctionals, unsaturated compounds, and aromatics. Their dataset was split into a training set of 310 reactions to derive the SAR and a test set of 124 reactions for validation. The large dataset enables a robust prediction of aqueous hydroxyl radical reaction rates with all organic compounds of atmospheric relevance. The authors were able to achieve a high accuracy and able to predict rate constants of $83 \%$ of the compounds in their training set and $62 \%$ of the compounds in their test set within a factor of 2 .

\subsubsection{SAR by Monod and Doussin}

The structure-activity relationship by Monod and Doussin (2008) covers the prediction of $\mathrm{OH}$ rate constants for alkanes, alcohols, carboxylic acids, and bases as well as polyfunctional compounds derived from these functions. A total of 72 compounds were used to derive the SAR. A major improvement of the SAR by Monod and Doussin (2008) is the introduction of a second correction factor for the incremental rate constants taking the effects of the $\beta$ neighbours into account. However, this improvement is also one of its biggest weaknesses as over-fitting becomes likely. With the dataset of 72 compounds, 22 descriptors have been defined. Moreover, a wide range of atmospherically relevant compounds is still missing, and currently only $\mathrm{H}$ abstraction reactions are possible to predict with this SAR. Yet high accuracy is achieved, and according to their own validation, Monod and Doussin (2008) predicted $60 \%$ of their compounds tested within the range of $80 \%$ of the experimental value.

The original SAR by Monod and Doussin (2008) was extended to include carbonyl compounds by Doussin and Monod (2013). The original parameters were kept constant, and new parameters have been introduced to describe the partial rate constant at aldehyde groups and $\alpha$ and $\beta$ effects of carbonyl (keto and aldehyde) groups. Moreover, a modulating factor for gem-diol groups in hydrated carbonyls was introduced.

\subsubsection{Comparison of both SARs}

In general, both SARs by Minakata et al. (2009) and Monod and Doussin (2008), with updates by Doussin and Monod (2013), are suitable tools for automated rate constant prediction of aqueous-phase hydroxyl radical reactions with organic compounds with the above-mentioned strengths and restrictions. The accuracy is high and implementation in 

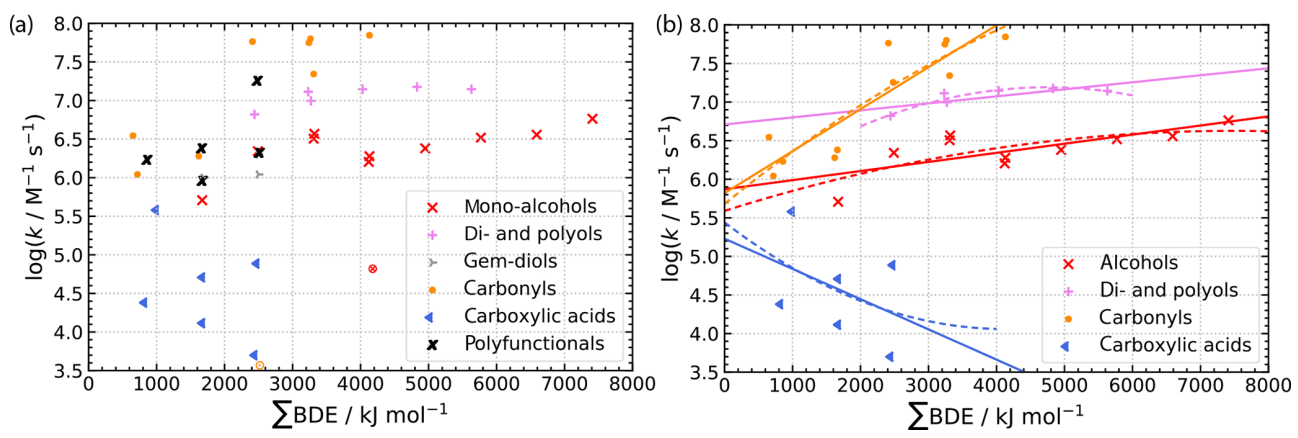

Figure 7. Experimental second-order rate constants versus accumulated bond dissociation enthalpies of all bonds in molecules containing $\mathrm{H}$ atoms ( $\Sigma$ BDE). Panel (a) shows the raw data and panel (b) the final datasets of the combined compound classes and their linear and quadratic regressions.

computer-assisted tools is easy. All parameters are delivered by the structure-activity relationships, and the only input variable is the chemical structure of the organic compound. Both SARs were tested using the kinetics database described in Sect. 2.1. They show a very good agreement with the experimental values, especially for simple molecules (see scatter plots in Fig. 8). Best results are achieved with pure hydrocarbons, and errors increase when introducing substituents. Largest errors occur for polyfunctional compounds. The box plots of the absolute errors of both SARs compared to the experimental values demonstrate these facts (see Fig. 9). More detailed information on the statistical data of the evaluation for every compound class can be found in the review by Herrmann et al. (2010).

The box plots in Fig. 9 show that both SARs have difficulties predicting the hydroxyl radical rate constants for dicarbonyl compounds (compound class 5). There is a large variation of about 3 orders of magnitude in the absolute errors between the minimum and the maximum value. Both SARs underpredict the rate constants. The difficulties may be caused by the small dataset of only five dicarbonyl compounds.

In general, errors are smaller in the SAR by Doussin and Monod (2013) due to the second descriptor for $\beta$ neighbours; however, it is noted again that over-fitting might be a problem in the SAR of these authors. Errors in both SARs are very small and besides a few exceptions well within 1 order of magnitude.

\section{Sensitivity runs of crucial parameters}

Based on the evaluation of the kinetic data and prediction methods for aqueous-phase rate constants, a preliminary protocol has been designed for mechanism auto-generation. The protocol uses SARs for hydroxyl radical reactions with organic compounds and the linear correlations of the refined Evans-Polanyi-type correlations for nitrate radical reactions with organic compounds. For uptake, the structure- activity relationship GROMHE (group contribution method for Henry's law estimate; Raventos-Duran et al., 2010) was used, and dissociations of carboxylic acids have been estimated with the method by Perrin et al. (1981). Fixed rate constants and branching ratios have been used for radical species. A more detailed description of all processes implemented in the protocol in its final state can be found in Table 2 in Sect. 4.

With the preliminary protocol, test mechanisms have been designed and evaluated focusing on the influence of critical parameters or the quality of estimates of parameters, where the scarcity of data allowed only rough estimates. All sensitivity studies have been performed with the parcel model SPACCIM (SPectral Aerosol Cloud Chemistry Interaction Model; Wolke et al., 2005) in a general, non-specific urban environment. Model runs are for $4.5 \mathrm{~d}$ at $45^{\circ} \mathrm{N}$ in mid-June. The trajectory of the parcel model involves eight cloud passages for about $2 \mathrm{~h}$ at noon and midnight of each day (marked by a blue shaded area in the results plots given in the following subsections). This scenario allows a detailed investigation of either particle or cloud chemistry and interactions between both (see Tilgner et al., 2013, for further information on the model scenario).

\subsection{Degradation of polycarbonyl compounds}

In a first sensitivity test, the formation of polycarbonyl compounds mostly in their hydrated form was observed in high concentrations. Diol functional groups were found at every site of the molecule, which is an unstable structure and should decompose thermally, and therefore, it should not exist in high concentrations. Hence, a decay of polycarbonyl compounds by $\mathrm{C}-\mathrm{C}$ bond breaking has been introduced to the protocol when at least three carbonyl groups (hydrated or unhydrated) are found within four adjacent carbon atoms. The rule is based on considerations in the gas-phase mechanism MCMv3.2 (Master Chemical Mechanism, http://mcm. leeds.ac.uk/MCMv3.2/, last access: 5 October 2015), where in the $n$-alkane oxidation series no more than three carbonyl 
(a) Alkanes and polyfunctionals

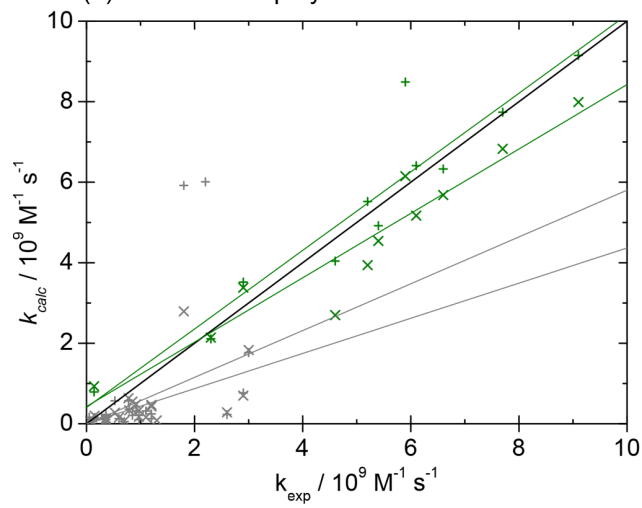

(c) Carbonyl compounds

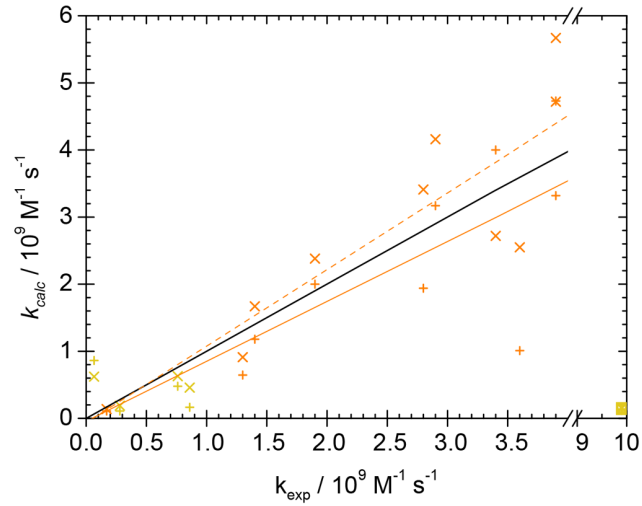

(b) Alcohol compounds

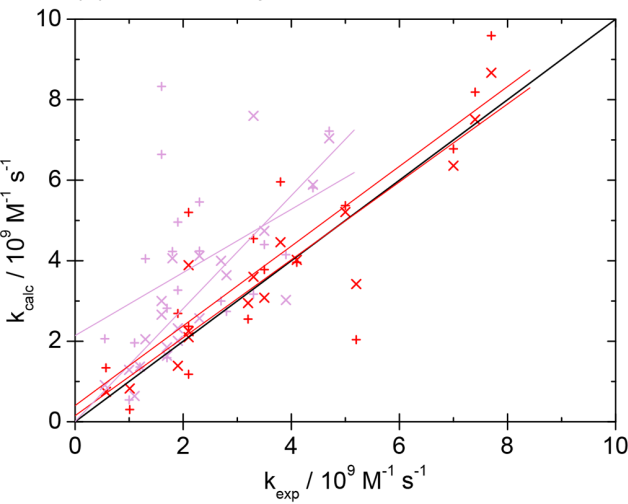

(d) Carboxylic acids

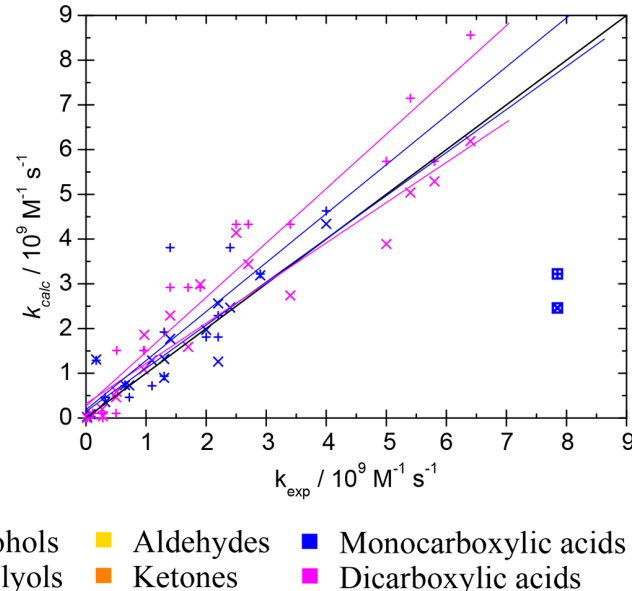

Figure 8. Scatter plots of predicted versus experimental data for the two SARs by Doussin and Monod (2013) and Minakata et al. (2009). Oblique crosses indicate predicted data by the SAR of Doussin and Monod (2013), with solid regression lines in the same colour. Upright crosses are data predicted with the SAR by Minakata et al. (2009), and dashed regression lines are in the same colour. The boxed value in panel (c) has been excluded as an outlier in the regressions. Regressions in panel (c) include both aldehyde and ketone data. Parameters for regression lines are given in Tables S13 and S14 in the ESM for the data by Doussin and Monod (2013) and Minakata et al. (2009), respectively.

groups are found within four adjacent carbon atoms independently of the chain length of the parent molecule.

For the estimation of the decay rate, sensitivity studies have been performed, where the first-order rate constant varied between 0.01 and $1 \mathrm{~s}^{-1}$. Moreover, the importance of $\mathrm{OH}$ radical induced oxidation of polycarbonyl compounds and the monomolecular decay has been investigated by allowing only molecular decay in the protocol or by treating both processes in parallel. The set of sensitivity studies was compared to the base case, which treats only the $\mathrm{OH}$ attack of polycarbonyl compounds. A short description of all sensitivity studies and of the nomenclature used in the following is given in Appendix A.2.

In the sensitivity runs, GECKO-A with a preliminary aqueous-phase protocol was used to extend CAPRAM 3.0n. The generator was used to revise the chemistry of all stable organic compounds in CAPRAM 3.0n and introduce branch- ing ratios for their degradation reactions. The preliminary protocol was applied to all newly evolving intermediates but was stopped at radical species, which were already previously defined in CAPRAM, to reduce the size of the mechanism. Besides a more detailed chemical scheme due to the treatment of minor reaction pathways in contrast to most reactions in the previous CAPRAM version, the hydration of carbonyl compounds is treated with greater complexity allowing the hydration of keto functionalities according to GROMHE.

Figure 10 shows the concentrations of 2-oxo-3-hydroxysuccinaldehyde (2O3H-SA), which illustrates the effects of the new protocol as it was already part of CAPRAM 3.0n. In this Figure and all other Figures, a " $\sum$ " before a species names or chemical formula in the unhydrated and protonated form means that the sum of the concentrations of all hydration and dissociation states of a given species are shown. Hy- 
Table 1. Parameterizations for the aqueous-phase chemistry of WSOC and HULIS compounds.

\begin{tabular}{|c|c|c|}
\hline Process & $k_{298} / K / j_{\max }^{\mathrm{a}}$ & Reference/comment \\
\hline $\mathrm{WSOC}+\mathrm{OH} \rightarrow \mathrm{WSOC}+\mathrm{HO}_{2}$ & $3.8 \times 10^{8}$ & Estimated after Arakaki et al. (2013) \\
\hline $\mathrm{HULIS}+\mathrm{OH} \rightarrow \mathrm{HULIS}+\mathrm{HO}_{2}$ & $3.8 \times 10^{8}$ & Estimated after Arakaki et al. (2013) \\
\hline HULIS $^{-}+\mathrm{OH} \rightarrow$ HULIS $^{-}+\mathrm{HO}_{2}$ & $3.8 \times 10^{8}$ & Estimated after Arakaki et al. (2013) \\
\hline $\mathrm{WSOC}+\mathrm{NO}_{3} \rightarrow \mathrm{WSOC}+\mathrm{HO}_{2}+\mathrm{NO}_{3}^{-}+\mathrm{H}^{+}$ & $1.0 \times 10^{7}$ & Estimated in accord with Sect. 3.3.1 \\
\hline HULIS $+\mathrm{NO}_{3} \rightarrow$ HULIS $+\mathrm{HO}_{2}+\mathrm{NO}_{3}^{-}+\mathrm{H}^{+}$ & $1.0 \times 10^{7}$ & Estimated in accord with Sect. 3.3.1 \\
\hline HULIS $^{-}+\mathrm{NO}_{3} \rightarrow$ HULIS $+\mathrm{HO}_{2}+\mathrm{NO}_{3}^{-}$ & $5.0 \times 10^{7}$ & Estimated in accord with Sect. 3.3.1 \\
\hline HULIS $\rightleftharpoons$ HULIS $^{-}+\mathrm{H}^{+}$ & $3.98 \times 10^{-4 \mathrm{~b}}$ & $\begin{array}{l}\text { Estimated with } p K_{\mathrm{a}} \text { of fulvic acid } \\
\text { after Salma and Láng (2008) }\end{array}$ \\
\hline $\mathrm{Fe}^{3+}+$ HULIS $^{-} \rightleftharpoons[\mathrm{FeHULIS}]^{2+}+0.5 \mathrm{HO}_{2}$ & $1.0 \times 10^{9 \mathbf{c}}$ & Estimated after iron-oxalate complex formation \\
\hline $\mathrm{Fe}^{2+}+$ HULIS $^{-} \rightleftharpoons\left[\mathrm{FeHULIS}^{+}\right.$ & $1.0 \times 10^{9 \mathrm{c}}$ & Estimated after iron-oxalate complex formation \\
\hline$[\text { FeHULIS }]^{2+} \stackrel{\text { hv }}{\longrightarrow} \mathrm{Fe}^{2+}+$ HULIS $+0.5 \mathrm{HO}_{2}$ & $2.0 \times 10^{-2}$ & $\begin{array}{l}\text { Estimated after iron-hydroxy complex } \\
\text { photolysis (see Arakaki et al., 2010) }\end{array}$ \\
\hline
\end{tabular}

${ }^{\text {a }} k_{298}$ is given for irreversible reactions (indicated by $\rightarrow$ ) in mole per litre and second $\left(\mathrm{M}^{-1} \mathrm{~s}^{-1}\right), K$ is given for equilibria (indicated by $\rightleftharpoons$ ) in mole per litre $\left(\mathrm{M}^{-1}\right)$. For photolysis (indicated by $\stackrel{\mathrm{hv}}{\longrightarrow}$ ), $j_{\max }$ is given in $\mathrm{s}^{-1}$. ${ }^{\mathrm{b}} k_{\text {backward }}=5.0 \times 10^{9} \mathrm{~s}^{-1} \cdot{ }^{\mathrm{c}} k_{\text {backward }}=3.0 \times 10^{-3} \mathrm{~s}^{-1}$.

dration is only considered in the sensitivity runs with the new protocol rules. Figure 10 shows that negligible concentrations are reached for the highly oxidized polycarbonyl compounds with rate constants of at least $0.1 \mathrm{~s}^{-1}$. Additional oxidation by hydroxyl radicals seems negligible as concentrations of the sensitivity runs with and without oxidation by $\mathrm{OH}$ radicals are indistinguishable from each other. However, product distributions are affected by the additional oxidation of $\mathrm{OH}$ radicals as can be seen from Fig. 11. The two acids shown in Fig. 11a and $b$ are the main degradation products of the OH-initiated oxidation of 2O3H-SA. While bond breaking reduces their concentrations compared to the base run with no monomolecular decay of polycarbonyls (woBB), this effect is counteracted in the runs with additional oxidation treated in the protocol especially for the run with the slow decay rate of $10^{-2} \mathrm{~s}^{-1}(\mathrm{BBe}-2+\mathrm{OH})$. Figure $11 \mathrm{c}$ and $\mathrm{d}$ show the concentration-time profiles of oxo-lactic and tartronic acid, the major first- and second-generation products of the monomolecular decay of $2 \mathrm{O} 3 \mathrm{H}-\mathrm{SA}$. It can be seen that the order of magnitude of the reaction rate constant has little effect on the concentrations of these products. Moreover, additional oxidation by hydroxyl radicals does not affect the formation of oxo-lactic acid and tartronic acid.

Finally, the implemented additional monomolecular decay of polycarbonyl compounds does not significantly increase the oxidation capacity of the aqueous phase. Macroscopic particle properties such as $\mathrm{pH}$ or organic particle mass are not affected by the additional decay channel. However, the high concentrations of highly oxidized and thermally unstable polycarbonyl compounds can be effectively suppressed with this new reaction type in the protocol. Therefore, it has been added to the final protocol. A decay rate of $0.1 \mathrm{~s}^{-1}$ was chosen as an ideal compromise between the suppression of high concentrations of polycarbonyls and an increased oxidation capacity of the aqueous phase. For complete descrip- tion in the final aqueous-phase protocol of GECKO-A, additional oxidation by hydroxyl radicals is considered as well to determine the product distribution with the best current knowledge.

\subsection{Influence of the chosen SAR}

SARs form the basis of the protocol for automated mechanism generation. Therefore, their influence on the oxidation mechanism and modelled concentrations of important constituents of the tropospheric aqueous phase has been investigated thoroughly. Figure S9 in the ESM shows the concentrations of selected organic compounds in the aqueous phase. Concentrations of compounds whose major source is the uptake from the gas phase are in excellent agreement for the two investigated SARs by Minakata et al. (2009) and Doussin and Monod (2013). However, significant differences in concentration-time profiles can be observed for secondor older-generation products mainly produced by aqueousphase processes (see Fig. S9).

This behaviour is explained by two facts. First, errors in the SARs increase with increasing complexity of the molecule and, hence, with more oxidized compounds in the aqueous phase. More importantly, this behaviour is a result of the excellent prediction of the overall rate constants by SARs in general, but there are significant uncertainties in the predicted products of aqueous-phase SARs due to missing experimental determinations of products and their branching ratios. Therefore, significant differences can be observed in the branching ratios while the rate constants of the overall decay compare well. More experimental data are needed to derive more reliable product distributions in aqueous-phase SARs. The macroscopic properties of particles such as $\mathrm{pH}$ and organic particle mass are not affected by these uncertainties (see Fig. S9 in the ESM), but significant differences 
Table 2. Processes implemented in the final GECKO-A aqueous-phase protocol for mechanism auto-generation.

\begin{tabular}{ll}
\hline Process & Estimation and/or comment \\
\hline Organic compounds $+\mathrm{OH}$ & SAR by Doussin and Monod (2013) accompanied by SAR by Minakata et al. (2009) for \\
& carboxyl or carboxylate groups and unsaturated carbon atoms with a double bond or groups \\
& in $\alpha$ and $\beta$ position to unsaturated carbon atoms. For organic nitrate functions the same group \\
& contribution factors for $\alpha$ and $\beta$ effects are assumed as for OH functions. Immediate oxygen \\
& addition is assumed to form alkyl radicals. $3 \%$ cut-off for minor branches.
\end{tabular}

Neutral saturated organic compounds Advanced Evans-Polanyi-type correlations using product determinations from the SAR of the $+\mathrm{NO}_{3}$ corresponding hydroxyl radical reaction.

Unsaturated organic compounds $+k_{2 \text { nd }}=1 \times 10^{7} \mathrm{M}^{-1} \mathrm{~s}^{-1}$ based on Sect. 3.3.2.

$\mathrm{NO}_{3}$

Dissociated carboxylic acids and $k_{2 \text { nd }}\left(\right.$ monocarboxylates $\left.+\mathrm{NO}_{3}\right)=2 \times 10^{7} \mathrm{M}^{-1} \mathrm{~s}^{-1}, k_{2 \text { nd }}\left(\mathrm{DCA}_{\text {mono-anion }}+\mathrm{NO}_{3}\right)=2.5 \times$ diacids $+\mathrm{NO}_{3}$ $10^{7} \mathrm{M}^{-1} \mathrm{~s}^{-1}$, and $k_{2 \text { nd }}$ (DCA dianion $+\mathrm{NO}_{3}$ ) $=7.75 \times 10^{7} \mathrm{M}^{-1} \mathrm{~s}^{-1}$ with ETR assumed as only reaction pathway and equal branching for unsymmetrical dicarboxylates. Nitrate radical addition to double bonds is assumed for unsaturated organic acids as the only reaction pathway instead of ETR with $k_{2 \mathrm{nd}}=1 \times 10^{7} \mathrm{M}^{-1} \mathrm{~s}^{-1}$.

Monomolecular decay of polycarbonyl Monomolecular decay of polycarbonyls with three carbonyl groups within four adjacent carcompounds bon atoms with $k_{1 \mathrm{st}}=0.1 \mathrm{~s}^{-1}$. Fragmentation is always between two carbonyl groups. Equal branching is assumed for unsymmetrical polycarbonyls with three adjacent carbonyl groups.

Hydrolysis of carbonyl nitrates Hydrolysis of nitrates with an adjacent carbonyl group is assumed with $k_{2 n d}=7.5 \times$ $10^{-6} \mathrm{M}^{-1} \mathrm{~s}^{-1}$ and $E_{\mathrm{A}} / R=6600 \mathrm{~K}$ based on PAN hydrolysis by Kames and Schurath (1995).

$\mathrm{RO}_{2} \stackrel{\mathrm{R}^{\prime} \mathrm{O}_{2}}{\longrightarrow} 0.4 \mathrm{RO}+1.1 \mathrm{R}=\mathrm{O}+0.5$ $\mathrm{ROH}+0.7 \mathrm{O}_{2}+0.3 \mathrm{H}_{2} \mathrm{O}_{2}$

Peroxy radical $\left(\mathrm{RO}_{2}\right)$ recombinations based on mechanism by von Sonntag and Schuchmann (1991) and von Sonntag et al. (1997) leading to alkoxy radicals (RO), carbonyl $(\mathrm{R}=\mathrm{O})$, and alcohol $(\mathrm{ROH})$ compounds with updated kinetic and mechanistic data for the acetonyl peroxy radical by Schaefer et al. (2012) using the given fixed branching ratios and $k_{2 \text { nd }}=7.3 \times 10^{8} \mathrm{M}^{-1} \mathrm{~s}^{-1}$.

Peroxy radicals with $\mathrm{OH}$ in $\alpha$ position $\quad \mathrm{HO}_{2}$ elimination assumed as only process with $k_{1 \mathrm{st}}=200 \mathrm{~s}^{-1}$. For a gem-diol function in $\alpha$ position, an increased $k_{1 \mathrm{st}}$ of $1000 \mathrm{~s}^{-1}$ is assumed.

$2 \mathrm{RCH}\left(\mathrm{O}_{2} \cdot\right) \mathrm{COOH} \longrightarrow 2 \mathrm{RCHO}+2$ Additional reaction pathway to recombination and cross-reactions for peroxy radicals with $\mathrm{CO}_{2}+\mathrm{H}_{2} \mathrm{O}_{2}$ $\mathrm{COOH} / \mathrm{COO}^{-}$in $\alpha$ position with estimated $k_{2 \mathrm{nd}}=200 \mathrm{~s}^{-1}$ leading to a carbonyl compound, $\mathrm{H}_{2} \mathrm{O}_{2}$, and dissolved $\mathrm{CO}_{2}$.

$2 \mathrm{RCH}_{\left(\mathrm{O}_{2}\right.} \cdot \mathrm{COO}^{-} \stackrel{2 \mathrm{H}_{2} \mathrm{O}}{\longrightarrow} \longrightarrow 2 \mathrm{RCHO}$
$+2 \mathrm{CO}_{2}+\mathrm{H}_{2} \mathrm{O}_{2}+2 \mathrm{OH}^{-}$

$\mathrm{RCO}_{3} \stackrel{\mathrm{H}_{2} \mathrm{O}}{\longrightarrow} \mathrm{RCOOH}+\mathrm{HO}_{2}$

Immediate hydration of acylperoxy radicals $\left(\mathrm{RCO}_{3}\right)$ and $\mathrm{HO}_{2}$ elimination with $k_{1 \mathrm{st}}=$ $1000 \mathrm{~s}^{-1}$ is assumed, leading to carboxylic acid formation ( $\left.\mathrm{RCOOH}\right)$.

Monomolecular decay of alkoxy radicals

$\mathrm{C}-\mathrm{C}$ bond breaking with equal branching for unsymmetrical molecules is assumed for alkoxy radicals with an estimated $k_{1 \mathrm{st}}$ of $5 \times 10^{2} \mathrm{~s}^{-1}$.

Alkoxy radicals $+\mathrm{O}_{2}$

Formation of $\mathrm{HO}_{2}$ and carbonyls with an estimated $k_{2 \mathrm{nd}}$ of $5 \times 10^{6} \mathrm{~s}^{-1}$.

Decomposition of acyloxy radicals

Decarboxylation by $\mathrm{C}-\mathrm{C}$ bond breaking of acyloxy radicals leading to dissolved $\mathrm{CO}_{2}$ and a peroxy radical (as immediate oxygen addition is assumed for the alkyl radical formed).

Hydration of carbonyl compounds

Hydration constants calculated with GROMHE (Raventos-Duran et al., 2010). Estimated $k_{\mathrm{b}}$ of $5.69 \times 10^{-3} \mathrm{~s}^{-1} ; k_{\mathrm{f}}$ calculated from $K$ and $k_{\mathrm{b}} .5 \%$ cut-off for minor hydration forms.

Dissociations of carboxylic acids

Calculated using $p K_{\mathrm{a}}$ prediction method with Taft parameters by Perrin et al. (1981) and an estimated backward rate constant of $5 \times 10^{10} \mathrm{M}^{-1} \mathrm{~s}^{-1} ; k_{\mathrm{f}}$ calculated from $p K_{\mathrm{a}}$ and $k_{\mathrm{b}}$. Currently no cut-off for minor dissociation states.

Phase transfer

HLC calculated using GROMHE; $D_{\mathrm{g}}$ calculated with FSG method (Fuller, 1986); $\alpha$ assumed 0.1 . 

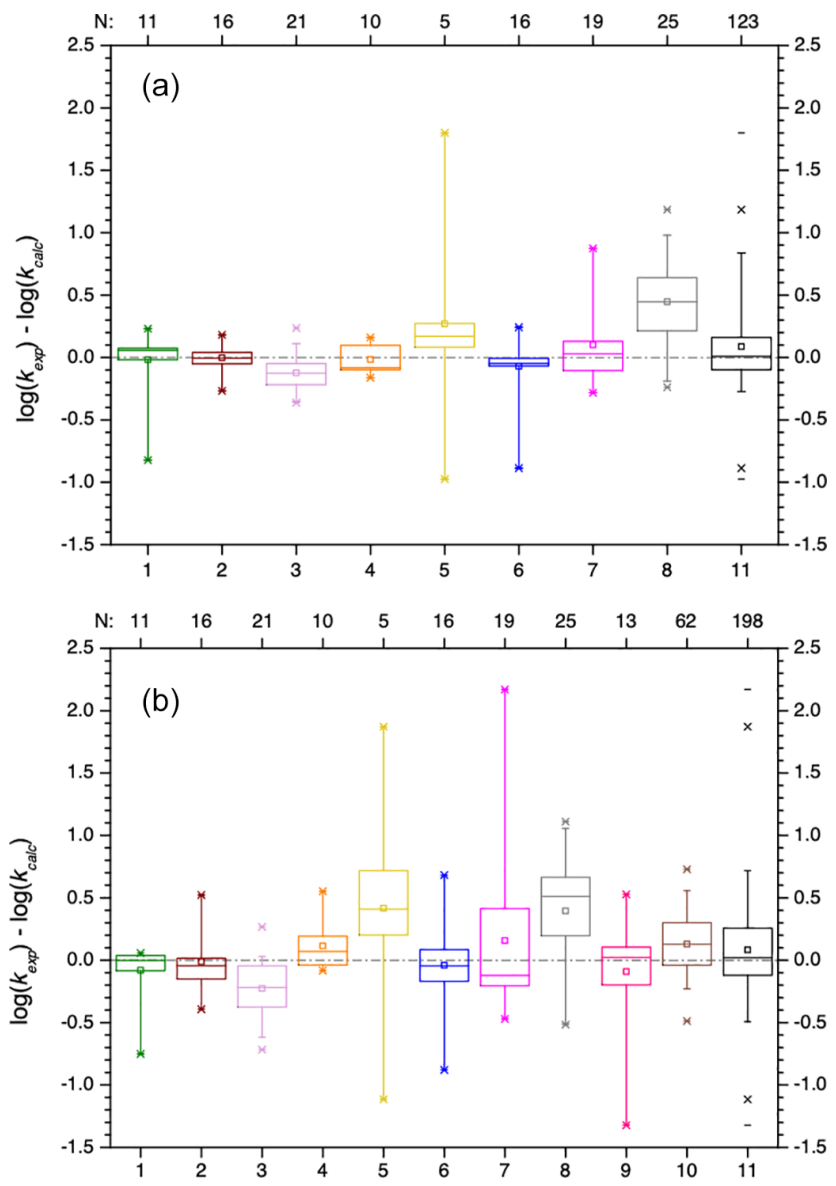

Figure 9. Box plots of the absolute errors of the logarithmized experimental versus predicted rate constants for the prediction with the SAR of Doussin and Monod (2013) (top) and Minakata et al. (2009) (bottom). Numbers at the abscissa refer to the compound class: 1 - alkanes, 2 - mono-alcohols, 3 - di- and polyols, 4 - carbonyls, 5 - dicarbonyls, 6 - mono-carboxylic acids, 7 - dicarboxylic acids, 8 - polyfunctionals, 9 - unsaturated compounds, 10 - aromatics, and 11 - all data.

in the prediction of the concentrations of single species might be observed between the two SARs.

\subsection{Processing of the organic mass fraction}

As the focus of this study is the aqueous-phase processing of organic particulate matter, a more detailed investigation has been performed for an urban environment with the improved mechanism from Sect. 3.1. From Fig. 12, two deficits of the current protocol can be seen. For such a polluted environment the growth of the organic mass fraction is too small. A significant mass increase of $166 \mu \mathrm{g} \mathrm{m}^{-3}$ is observed in the base model run as seen in Fig. S10 of the ESM, while the organic mass increases by only $1.7 \mu \mathrm{g} \mathrm{m}^{-3}$ contributing less than $5 \%$ to the total mass at the end of the model run. This is significantly less than observed all around the world (Jimenez et al., 2009). The organic mass increase is signif-

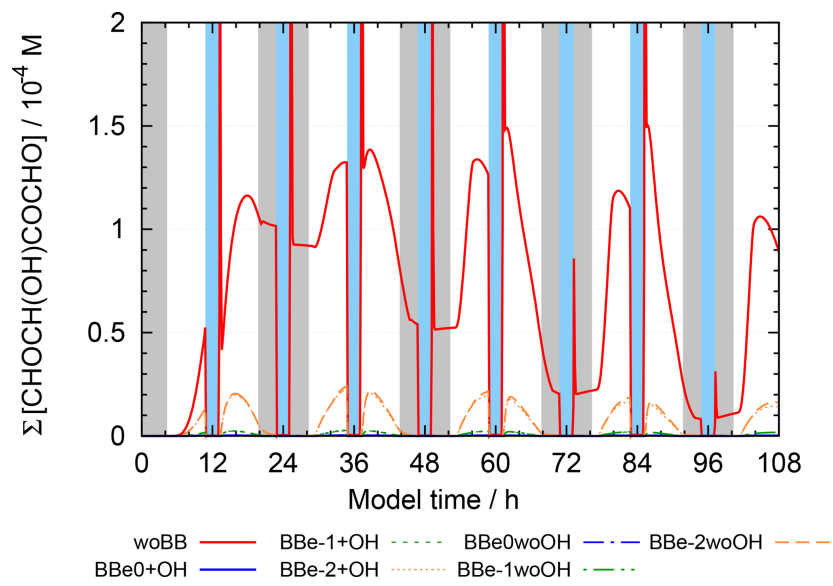

Figure 10. Concentration-time profiles of aqueous 2-oxo-3hydroxy-succinaldehyde in all hydration forms in the sensitivity runs investigating the decay of polycarbonyl compounds under urban summer conditions with eight cloud passages using the preliminary CAPRAM-GECKO-A protocol described in the text. Cloud passages are shaded blue and night-time periods are shaded grey. Scenario names used in the legend are explained in Appendix A.2.

icantly less than observed previously (Brégonzio-Rozier et al., 2016; Ervens and Kreidenweis, 2007). Secondly, unrealistically high concentrations of organic nitrates are observed during non-cloud periods. During non-cloud periods, the majority $(>80 \%)$ of the particle mass of the explicit scheme (excluding the generic model species humic-like substances, HULISs; water-soluble organic compounds, WSOCs; and water-insoluble organic compounds, WISOCs) consists of organic nitrates. In general, organic nitrate fractions of up to $20 \%$ are observed (Day et al., 2010) and up to $45 \%$ are observed in submicron aerosol and particulate matter of aerosol particles with a diameter $\leq 1 \mu \mathrm{m}$ (PM1) (Bean et al., 2016; Kiendler-Scharr et al., 2016). To address these issues, further investigations were performed on the processing of the organic particulate matter with a focus on organic nitrates. A series of test studies were performed, which led to a significant improvement of the description of the processing and the composition of organic particulate matter. These studies are described in the following subsections. The best estimates of each test scenario are taken over and included in the following model runs unless stated otherwise.

\subsubsection{Proxy reactions for $\mathrm{OH}$ and $\mathrm{NO}_{3}$ radical reactions with residual $\mathrm{OM}$}

Despite its great level of detail, the mechanism generated by the preliminary protocol for these sensitivity studies is still limited as it only treats the chemistry of compounds with up to four carbon atoms explicitly. Therefore, radical concentrations might be overestimated due to missing scavenging by larger organic compounds, which further affects important particle properties and particle growth. Therefore, 
(a) 3,4-Dioxo-2-hydroxy-butyric acid

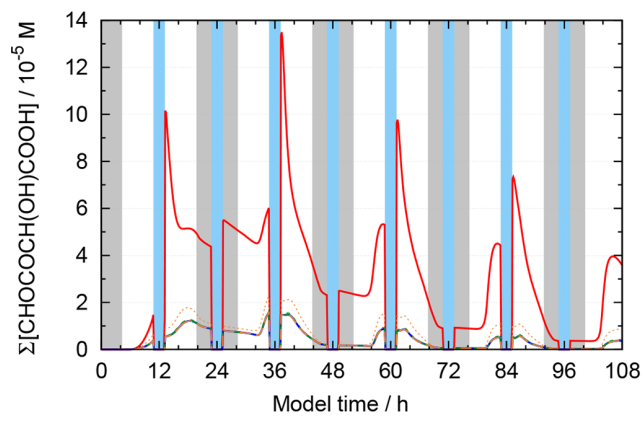

(c) Oxo-lactic acid

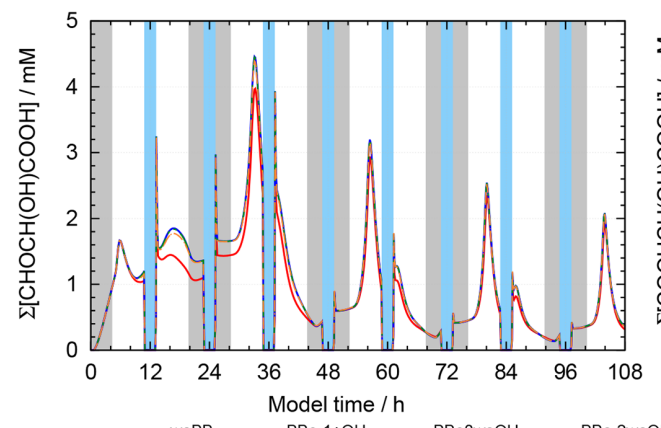

(b) 2,4-Dioxo-3-hydroxy-butyric acid

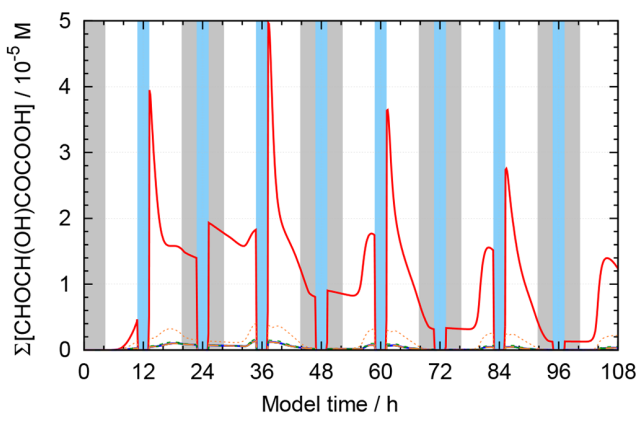

(d) Tartronic acid

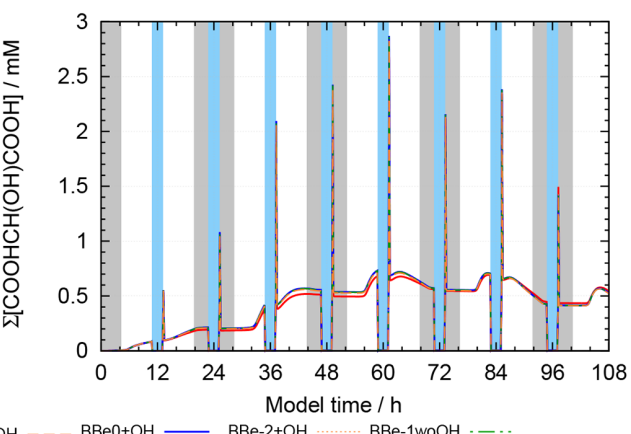

Figure 11. Accumulated concentration-time profiles of all hydration and dissociation forms of products of 2-oxo-3-hydroxy-succinaldehyde in the sensitivity runs investigating the decay of polycarbonyl compounds under urban summer conditions with eight cloud passages using the preliminary CAPRAM-GECKO-A protocol described in the text. Cloud passages shaded blue and night-time periods are shaded grey. Scenario names used in the legend are explained in Appendix A.2.

proxy reactions for the processing of the generic watersoluble organic compounds (WSOCs) and humic-like substances (HULISs or HULISs ${ }^{-}$) have been introduced to CAPRAM 3.0n to account for the interactions of hydroxyl and nitrate radicals with longer-chained organic compounds. The generic model species WSOCs, HULISs or HULISs ${ }^{-}$as well as water-insoluble organic compounds (WISOCs) are referred to as residual organic matter (residual $\mathrm{OM}$ ) in the following. All proxy reactions are given in Table 1. Besides the oxidation by $\mathrm{OH}$ and $\mathrm{NO}_{3}$, photolysis of humic-like substances is included to account for an accurate description of the iron(II) to iron(III) ratio.

The new proxy reactions decrease radical oxidant concentrations as expected (see Fig. S11 in the ESM for $\mathrm{OH}$ and $\mathrm{NO}_{3}$ concentrations in the run WSOCs). Daytime $\mathrm{OH}$ radical concentrations are decreased by more than 1 order of magnitude and nitrate radical concentrations by almost 1 order of magnitude by the end of the simulation after 4.5 simulated days. Reduced radical oxidant concentrations cause an increase in organic particulate matter as a result of decreased sinks and equal sources of the dissolved organic matter compared to previous model runs. Figure 13 illustrates the mass increase showing the total organic particle mass for the scenarios with (WSOCs) and without (orig) reactions of radical oxidants with the residual OM as well as the organic nitrates and the residual OM fraction. As the sensitivity runs focus on non-cloud periods, high concentrations during cloud periods are not shown in Fig. 13 or any other figures of this subsection for a better resolution of the differences during non-cloud periods.

Figure 13 shows that previously particle growth was observed only during cloud periods, while the organic mass during non-cloud periods decreased. With decreased $\mathrm{OH}$ and $\mathrm{NO}_{3}$ radical concentrations from the WSOC and HULIS proxy reactions less oxidation is observed. The reason for this behaviour is that the current protocol consists mainly of fragmentation reactions, which produce mainly highvolatility products. Thus, the decreased oxidation in the WSOCs' runs suppresses the production of volatile organic compounds and reduces their release to the gas phase. As a consequence, the observed decrease in organic particle mass during non-cloud periods is less with the proxy reactions for WSOC and HULIS species.

These sensitivity runs also demonstrate a weakness of the current protocol, which only includes oxidative processes. Non-oxidative processes, such as accretion reactions are currently not considered. This is partly due to very limited experimental data and partly due to the fact that non-oxidative processes will rapidly lead to very large products, which require large explicit oxidation schemes. With the currently 


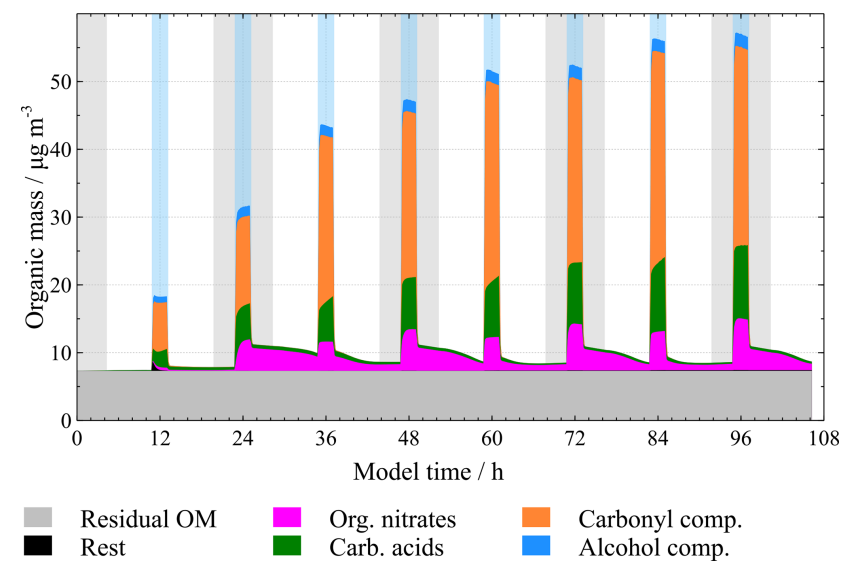

Figure 12. Particulate organic mass fraction distinguished by constituents in the base scenario "orig" using the preliminary mechanism from Sect. 3.1 under urban summer conditions with eight cloud passages. Cloud passages are shaded blue and night-time periods are shaded grey.

very comprehensive protocol, the mechanism size would increase beyond the capabilities of current numeric models. Furthermore, fragmentation seems overestimated in the current protocol, a fact that is addressed in another sensitivity study introduced in Sect. 3.3.3. While the new proxy reactions help to increase organic particle growth rates to more realistic values, they do not affect particle composition. Therefore, the overestimation of organic nitrate concentrations remains, and further tests are introduced in the next subsection to investigate the formation and degradation of organic nitrates and to identify missing processes in the current protocol.

\subsubsection{Detailed studies of organic nitrate sinks and sources}

Further tests have been performed on the sinks and sources of organic nitrates to investigate the cause of their high concentrations in the model simulations. In the current protocol, photolysis is excluded due to the very limited data, which makes it hard to determine rules for automated prediction of photolysis rates. To evaluate effects on the processing of organic nitrates in the aqueous phase, photolysis has been included for this compound class leading to alkoxy radicals and $\mathrm{NO}_{2}$.

$\mathrm{RONO}_{2} \longrightarrow \mathrm{RO}+\mathrm{NO}_{2}$

In the estimation of a maximum photolysis efficiency, the photolysis rate was estimated twice as high as gas-phase photolysis of typical organic nitrates. This estimate recognizes the lens effects deriving from the spherical shape of cloud droplets and particles (e.g. Mayer and Madronich, 2004), but it fully neglects any solvent cage effects. Hence, photolysis processes of organic nitrates in aqueous solution must be

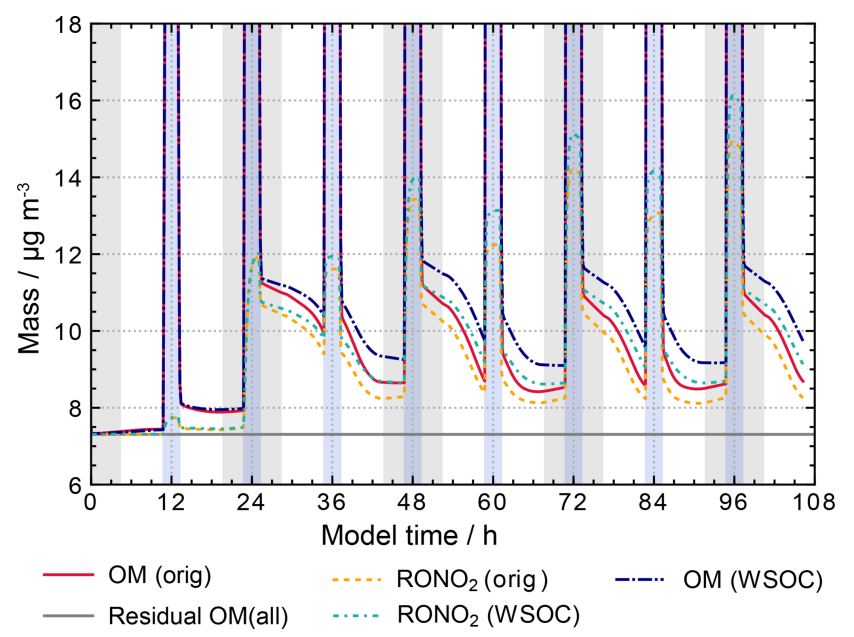

Figure 13. Processing of the organic mass fraction in the sensitivity runs investigating the influence of parameterizations for WSOC chemistry and HULIS chemistry on aqueous-phase chemistry and composition using the same conditions as in the base scenario with additional reactions given in Table 4 . Cloud passages are shaded blue and night-time periods are shaded grey. Scenario names used in the legend are explained in Appendix A.2.

considered as an upper-limit estimate. For neutral photofragments, effective quantum yields similar to their gas-phase counterparts have been discussed before (Herrmann, 2007).

Only minor effects from additional photolysis of organic nitrates (even using the upper-limit estimates) are observed in the scenario "Phot" compared to scenario "WSOC" (see Fig. 14). Organic nitrates remain the dominant fraction and their reduction is negligible. Therefore, photolysis for organic nitrates has been neglected in the final protocol (and the following sensitivity studies) to be more consistent with the other compound classes and until the development of a more vigorously tested photolysis protocol for all chromophores.

Further investigation focused on possible overestimations of the sources of organic nitrates. The only aqueous-phase source of organic nitrates, besides possible uptake from the gas phase, is the addition of nitrate radicals to double bonds of unsaturated organic compounds. Therefore, a sensitivity study has been performed with reduced rate constants of the addition reaction. In the original protocol, different estimates for different compound classes were made based on experimental determinations by Alfassi et al. (1993) as given in Table S2 in the ESM. Their measurements indicate high reaction rate constants of the order of $10^{9} \mathrm{M}^{-1} \mathrm{~s}^{-1}$ for unsaturated compounds and rate constants of the order of $10^{7} \mathrm{M}^{-1} \mathrm{~s}^{-1}$ for carboxylic acids. However, recent measurements by Schöne et al. (2014) indicate reduced rate constants for unsaturated compounds. Therefore, rate constants of unsaturated compounds with nitrate radicals were restricted to $10^{7} \mathrm{M}^{-1} \mathrm{~s}^{-1}$ in another sensitivity study labelled with "NO3add" in Fig. 14. 


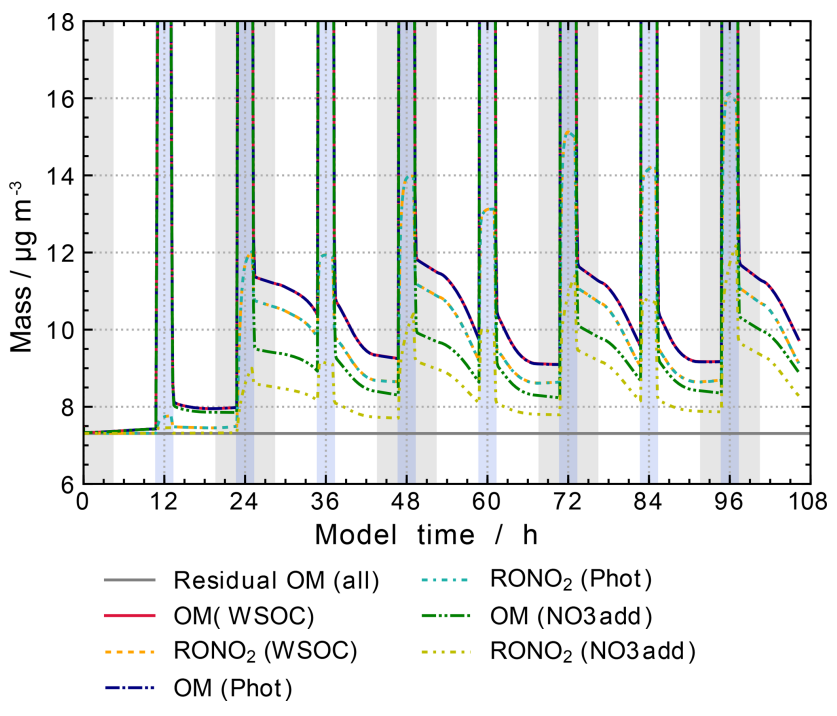

Figure 14. Processing of the organic mass fraction in the sensitivity runs investigating the influence of sink and source reactions for organic nitrates on aqueous-phase chemistry and composition. In addition to the scenario "WSOC", rate constants of all $\mathrm{NO}_{3}$ reactions with unsaturated organic compounds have been set to $10^{7} \mathrm{M}^{-1} \mathrm{~s}^{-1}$ in scenario "NO3add", and photolysis reactions with $j=3.6 \times 10^{-7} \mathrm{~s}^{-1}$ have been added in scenario "Phot". Cloud passages are shaded blue and night-time periods are shaded grey. Scenario names used in the legend are explained in Appendix A.2.

The reduction of the rate constants of $\mathrm{NO}_{3}$ addition reactions significantly reduces the mass fraction of organic nitrates but consequently also suppresses particle growth (see scenario "NO3add" in Fig. 14). The described attempts are unable to resolve the opposing effects of overestimated organic nitrate concentrations and underestimated particle growth. Therefore, further investigations were performed focusing on the phase transfer in the aqueous-phase protocol of GECKO-A.

\subsubsection{Phase transfer of oxygenated organic compounds}

GECKO-A uses the structure-activity relationship GROMHE (Raventos-Duran et al., 2010) to estimate the Henry's law coefficients (HLCs) needed to describe the uptake process. However, recent research indicates that due to the data used to derive the SAR, HLCs might be underestimated in GROMHE (Compernolle and Müller, $2014 \mathrm{~b}$, a). To address a possible overestimated release of organic particulate constituents to the gas phase, a sensitivity study ("O : $\mathrm{C}=1$ ") has been performed, where the HLC of all compounds with an $\mathrm{O}: \mathrm{C}$ ratio greater than or equal to 1 has been set to a fixed value of $1 \times 10^{9} \mathrm{M} \mathrm{atm}^{-1}$.

The results are shown in Fig. 15. The new estimates increase the organic mass concentration during non-cloud periods to values between 9 and $13 \mu \mathrm{g} \mathrm{m}^{-3}$. A noticeable difference is the faster particle growth during non-cloud peri-

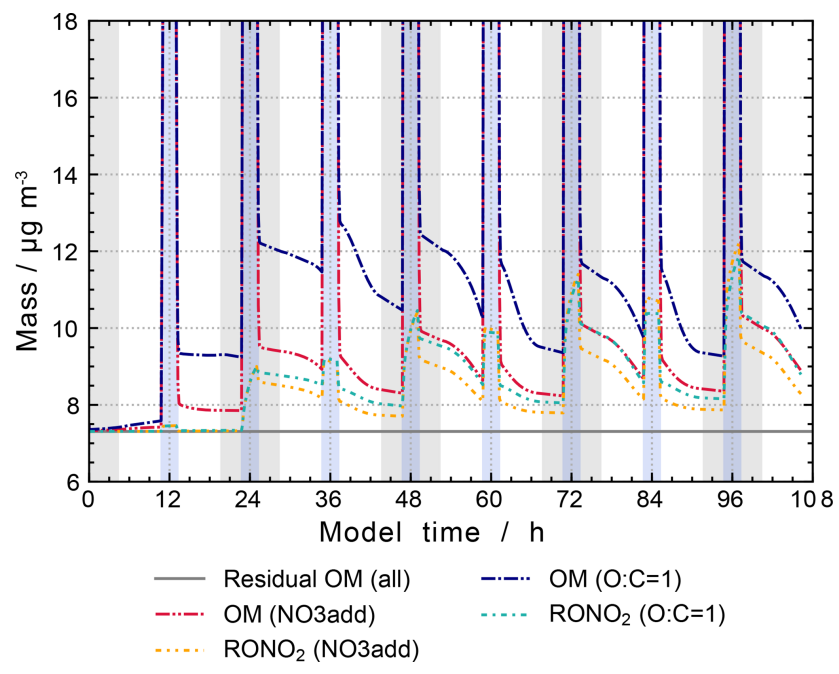

Figure 15. Processing of the organic mass fraction in the sensitivity runs investigating the influence of the phase transfer of oxygenated organic compounds on aqueous-phase chemistry and composition. The same conditions as in the scenario "NO3add" were used but with HLCs set to $1 \times 10^{9} \mathrm{M} \mathrm{atm}^{-1}$ for species with an $\mathrm{O}: \mathrm{C}$ ratio greater than or equal to 1 . Cloud passages are shaded blue and nighttime periods are shaded grey. Scenario names used in the legend are explained in Appendix A.2.

ods in the model run with the revised uptake, reaching the (non-cloud) peak value of $13 \mu \mathrm{g} \mathrm{m}^{-3}$ directly after the daytime cloud periods on the second model day. Thereafter, peak values after cloud periods are unchanged or slightly decreasing. This is in contrast to the model runs that use only the GROMHE estimates, where a smaller particle growth is observed, however, with constantly increasing peak values after cloud periods. The new estimates lead to an increase in organic particulate mass of $2 \mu \mathrm{g} \mathrm{m}^{-3}$ at the end of the model run.

Other studies have previously shown that different estimation methods of HLCs lead to important differences of several orders of magnitude (Wang et al., 2017). The current deficits can only be overcome with further development of the estimation methods for HLCs using an updated database of experimentally determined Henry's law coefficients. For the current study, HLCs of species with an $\mathrm{O}: \mathrm{C}$ ratio larger than or equal to 1 are set to $1 \times 10^{9} \mathrm{M} \mathrm{atm}^{-1}$. However, uptake processes are currently the source of one of the largest uncertainties in the protocol.

\subsubsection{Decay of alkoxy radicals}

Further investigation focused on the chemistry of alkoxy radicals in the database. Previous CAPRAM estimates derived from the MOST Project (George et al., 2005) considered two 
degradation pathways with the following rate constants:

$$
\begin{aligned}
& \mathrm{R}_{1} \mathrm{R}_{2} \mathrm{O}^{\cdot} \longrightarrow \mathrm{R}_{1}+\mathrm{R}_{2}=\mathrm{O}\left(5 \times 10^{5} \mathrm{~s}^{-1}\right), \\
& \mathrm{RO}^{\circ}+\mathrm{O}_{2} \longrightarrow \mathrm{R}=\mathrm{O}+\mathrm{HO}_{2}\left(5 \times 10^{6} \mathrm{M}^{-1} \mathrm{~s}^{-1}\right) .
\end{aligned}
$$

With typical modelled aqueous-phase oxygen concentrations of 3.5-4.0 $\times 10^{-4} \mathrm{M}$, this means that almost all alkoxy radicals decay by $\mathrm{C}-\mathrm{C}$ bond breaking. Here, the pseudo firstorder reaction rate constant of the oxygen channel is about a factor of 250 smaller. Therefore, this estimate favours fragmentation over the oxidation to more polar products, with an increased $\mathrm{O}: \mathrm{C}$ ratio whose further oxidation can potentially lead to highly soluble multifunctional compounds. Hence, the degradation reaction has been re-investigated by varying the decay rate of the fragmentation channel over 5 orders of magnitude from $5 \times 10^{0}$ to $5 \times 10^{5} \mathrm{~s}^{-1}$. The results are shown in Fig. 16. As expected, a big increase in particulate matter is observed in the sensitivity run with a decay rate of $5 \times 10^{2} \mathrm{~s}^{-1}$, where the ratio of the rate constant of the fragmentation channel to the pseudo first-order rate constant of the oxygen channel decreases to 0.25 . With the competitiveness of the oxygen channel, particle growth increases by about $3 \mu \mathrm{g} \mathrm{m}^{-3}$ in $4.5 \mathrm{~d}$ (see Fig. 16). Further reductions of the rate constant of the monomolecular decay channel affect particle growth less, leading to the production of an additional $1 \mu \mathrm{g} \mathrm{m}^{-3}$ at the end of the model run. Therefore, $5 \times 10^{2} \mathrm{~s}^{-1}$ has been used as the best estimate for the degradation channel in the final protocol. However, measurements of the decay of alkoxy radicals are urgently needed for a wider range of compounds and, in particular, for the ratio of the different product channels. The current mechanism is based on just two measurements of aliphatic alkoxy radicals - the methoxy radical (Schuchmann and von Sonntag, 1984) and the ethoxy radical (Bonifaciić et al., 2003).

\subsection{Influence of nitrate radical chemistry}

The explicit description of nitrate radical chemistry can lead to an increase in the size of the generated mechanisms, in particular, when unsaturated organic compounds are involved. Unsaturated compounds preferably react by addition reactions with the attacking radicals. Therefore, organic nitrates are formed in reactions of $\mathrm{NO}_{3}$ radicals with unsaturated compounds, which were not treated in previous CAPRAM mechanisms. For each new organic nitrate, a complete oxidation scheme has to be generated up to $\mathrm{CO}_{2}$. Only one reaction type has been implemented to remove the nitrate group from the carbon skeleton: the hydrolysis of carbonyl nitrates, which was estimated in accordance with peroxyacyl nitrate (PAN) hydrolysis.

$\mathrm{RC}(=\mathrm{O}) \mathrm{ONO}_{2} \stackrel{\mathrm{H}_{2} \mathrm{O}}{\longrightarrow} \mathrm{RC}(=\mathrm{O}) \mathrm{OH}+\mathrm{NO}_{3}^{-}+\mathrm{H}^{+}$

However, with estimated rate constants of $k_{2 \text { nd }}=7.6 \times$ $10^{-6} \mathrm{M}^{-1} \mathrm{~s}^{-1}$ and $E_{\mathrm{A}} / R=6600 \mathrm{~K}$ (Kames and Schurath,

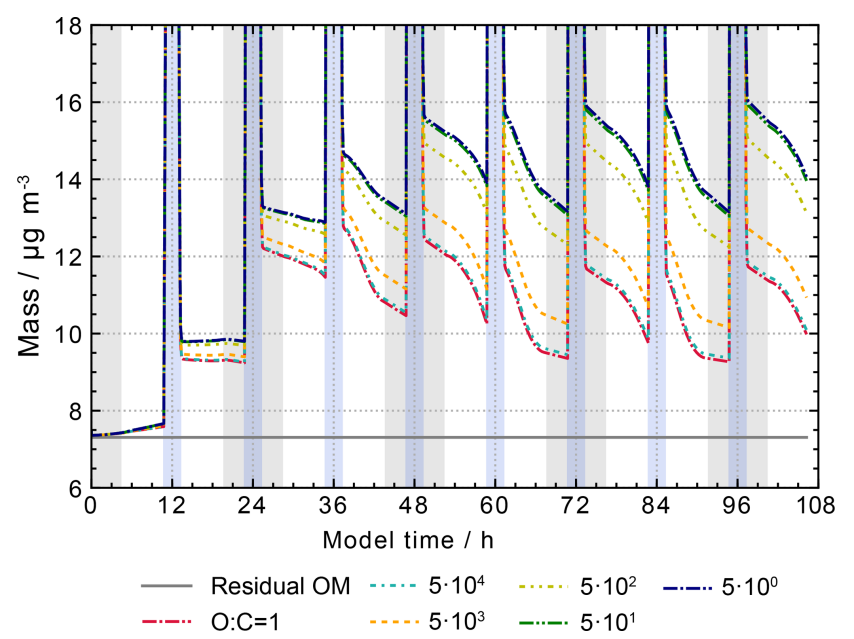

Figure 16. Processing of the organic mass fraction in the sensitivity runs investigating the influence of alkoxy radical chemistry on aqueous-phase chemistry and composition using the same conditions as in the scenario with $\mathrm{O}: \mathrm{C}=1$ but varying the monomolecular decay rate of alkoxy radicals as given in the figure legend as per second $\left(\mathrm{s}^{-1}\right)$. Cloud passages are shaded blue and night-time periods are shaded grey.

1995) from PAN hydrolysis, this process is very slow and only important for highly oxidized species, which possess no carbon bound $\mathrm{H}$ atoms and therefore cannot be oxidized by radical attack. Therefore, the nitrate function is likely to remain in the molecular structure throughout the whole oxidation process to small molecules. Hence, a large additional reaction scheme is needed. The sensitivity runs in Sect. 3.3 have demonstrated the need to include these reactions in polluted environments. However, in clean environments or for chamber modelling under low-NOx conditions, such detailed chemistry might not be needed. Therefore, GECKO-A has been equipped with three options to treat nitrate radical chemistry. For every chemical mechanism, three levels of reduction are generated.

- The $\alpha$ version: treatment of complete nitrate radical chemistry. Full mechanism generated.

- The $\beta$ version: reactions with unsaturated compounds prohibited to suppress organic nitrate formation. $\mathrm{H}$ atom abstraction reactions are allowed with saturated compounds but not with unsaturated compounds to prevent a shift in the reaction products.

- The $\gamma$ version: no nitrate radical chemistry allowed at all, except for the chemistry already used in previous CAPRAM versions.

Sensitivity studies have been performed under urban and remote conditions, which confirm the importance of nitrate radical chemistry in highly polluted regimes and the necessity for a complete treatment of these compounds as stressed 
in Sect. 3.3. Under remote conditions, organic nitrate formation is negligible with a total organic nitrate mass of less than $5 \times 10^{-12} \mathrm{~g} \mathrm{~m}^{-3}$. Therefore, it is safe to use the $\beta$ version of the generated mechanism in these environments. The significance of nitrate radical chemistry is low in general in these environments according to the model simulations. Differences in the concentration-time profiles between the $\beta$ and the $\gamma$ versions are only observed for highly oxidized dicarboxylic acids, where electron transfer dominates over $\mathrm{H}$ abstraction reactions. Results for these remote conditions are presented for selected organic compounds in Fig. S12 in the ESM. Therefore, for pristine environments or very clean chamber conditions, the $\gamma$ version of the mechanism might be suitable as well; however, errors increase especially for highly oxidized organic compounds. Moreover, the potential for mechanism reduction is much smaller for the omission of $\mathrm{H}$ abstraction reactions as generally the same products are produced in these reactions as are produced for the hydroxyl radical attack. Therefore, the generator does not create new reaction schemes for new intermediate compounds and the mechanisms are only reduced for the nitrate radical reactions.

\subsection{Investigation of further parameters in the protocol}

Further sensitivity studies have been employed to investigate the sensitivity of the generated mechanisms towards the mass accommodation coefficient $\alpha$ in the uptake process and the choice of the threshold percentage to omit minor reaction pathways. These results are outlined here only in brief.

\subsubsection{Uptake parameters}

Only very few experimental determinations of mass accommodation coefficients exist in the literature (see, e.g. the most recent IUPAC recommendation by Ammann et al., 2013; or the compilations by Davidovits et al., 2006, 2011). Therefore, it is impossible to derive advanced estimation methods for this parameter. Previous CAPRAM and GECKO-A (Mouchel-Vallon et al., 2013) estimates used a fixed estimate of 0.1. To evaluate the sensitivity of this parameter, different model runs with varying mass accommodation coefficient estimates from the lowest value in the CAPRAM database of 0.0067 for methyl hydroperoxide to the highest value of 0.5 for $\mathrm{HONO}$ were used.

No effects on any concentration-time profile could be detected. Therefore, this parameter seems insensitive in the typical range found for organic compounds relevant for tropospheric multiphase chemistry, and the previous CAPRAM estimate of 0.1 has been taken over in the GECKO-A protocol.

\subsubsection{Cut-off thresholds}

Furthermore, thresholds for the cut-off of minor reaction pathways have been investigated as a means of mechanism reduction. In the aqueous-phase protocol of GECKO-A, a choice of different cut-off parameters is possible. The generator also ensures that the omission of minor reaction pathways does not result in the loss of too many reaction pathways as described in detail in the next section. As shown in Fig. S13 in the ESM, the number of reduced reactions increases linearly with the percentage of the cut-off parameter. Significant differences occur mainly for very crude thresholds of $10 \%$ or higher (see Fig. S14 in the ESM). In the current protocol, a $3 \%$ threshold was chosen as standard value to compromise between a most detailed description of the chemistry in benchmark mechanisms and a reduced mechanism size for better numerical processing.

\section{The new multiphase mechanism generator GECKO-A}

Based on the evaluation of kinetic data and prediction methods in Sect. 2 and the sensitivity studies in Sect. 3, a protocol for aqueous-phase mechanism auto-generation has been defined. The final protocol is described in Sect. 4.1. Its implementation in CAPRAM-GECKO-A and the workflow of the new multiphase mechanism generator are explained in Sect. 4.2.

\subsection{The new aqueous-phase protocol for GECKO-A}

This section details the rules defined to predict mechanistic, kinetic, and thermodynamic data for mechanism autogeneration in GECKO-A. An overview of all processes in the new aqueous-phase protocol, and the estimates used to derive rate constants, further kinetic, and thermodynamic parameters are given in Table 2 .

SARs form the basis of the protocol due to the highest accuracy and the ease to implement them in automated computer tools. Furthermore, a major advantage over all other estimation methods is the ability to predict reaction products. In the current protocol, the SAR by Doussin and Monod (2013) is preferred over the SAR by Minakata et al. (2009). Despite a similarly successful generation of overall rate constants, attempts have been made to acknowledge branching ratios from measurements by Asmus et al. (1973) in the SAR by Monod and Doussin (2008) (Anne Monod, personal communication, 15 April 2010). Therefore, the SAR was chosen for a best possible description of the product distribution. In the protocol, the SAR is supplemented with parameters from the SAR by Minakata et al. (2009) for unsaturated compounds or $\mathrm{H}$ atom abstraction reactions on carboxyl groups, which is neglected in the SAR by Monod and Doussin (2008) due to the very low partial rate constants. Hence, it is possible to have a mixture of different SARs used to predict the rate constant for one compound. In the generator, molecules are treated group-wise for every carbon atom bearing group. Where possible, the SAR by Doussin and Monod (2013) is used and only those groups are supplemented with the SAR 
Table 3. Measured uncorrected concentrations in micrograms per cubic metre $\left(\mu \mathrm{g} \mathrm{m}^{-3}\right)$ from C-GIS and the PTFE filter samples for identified products. Times given in the first column refer to measurement points in hours and minutes after the injection of ozone and the start of the UV-C photolysis.

\begin{tabular}{|c|c|c|c|c|c|c|c|c|c|}
\hline Sample & Time & Methylglyoxal & Formate & Acetate & Pyruvate & Oxalate & Malonate & Succinate & Malate \\
\hline \multicolumn{10}{|c|}{ C-GIS samples (times correspond to the start of experiment, sample A00 is used to correct for background chemistry) } \\
\hline $\mathrm{A} 00^{\mathrm{a}}$ & $-00: 27$ & - & 0.230 & 0.395 & - & 0.199 & 0.019 & 0.049 & 0.422 \\
\hline $\mathrm{A} 01^{\mathrm{b}}$ & 00:01 & - & 0.328 & 0.512 & - & 0.212 & 0.016 & 0.105 & 0.748 \\
\hline A02 & $00: 16$ & - & 0.266 & 0.433 & - & 0.213 & 0.022 & 0.074 & 0.475 \\
\hline A03 & 00:31 & - & 0.340 & 0.783 & - & 0.355 & 0.046 & 0.130 & 0.475 \\
\hline A04 & $00: 46$ & - & 0.159 & 0.427 & - & 0.178 & 0.017 & 0.048 & 0.149 \\
\hline A05 & 01:01 & - & 0.026 & 0.630 & - & 0.185 & 0.014 & 0.150 & 0.004 \\
\hline A06 & 01:16 & - & 0.230 & 0.570 & - & 0.237 & 0.018 & 0.116 & 0.083 \\
\hline A07 & 01:31 & - & 0.137 & 0.436 & - & 0.189 & 0.018 & 0.044 & 0.052 \\
\hline A08 & 01:46 & - & 0.230 & 0.798 & - & 0.315 & 0.016 & 0.023 & 0.055 \\
\hline A09 & 02:01 & - & 0.374 & 1.469 & - & 0.651 & 0.043 & 0.087 & 0.079 \\
\hline A10 & $02: 16$ & - & 0.230 & 0.887 & - & 0.355 & 0.031 & 0.058 & 0.026 \\
\hline A11 & $02: 31$ & - & 0.477 & 2.169 & - & 0.824 & 0.039 & 0.070 & 0.064 \\
\hline A12 & $02: 46$ & - & 0.170 & 0.908 & - & 0.260 & 0.037 & 0.098 & 0.027 \\
\hline A13 & 03:01 & - & 0.307 & 1.798 & - & 0.474 & 0.028 & 0.021 & 0.018 \\
\hline A14 & $03: 16$ & - & 0.362 & 2.194 & - & 0.703 & 0.043 & 0.087 & 0.037 \\
\hline \multicolumn{10}{|c|}{ PTFE filter samples (collected after the end of the experiment at 03:30 h) } \\
\hline $\mathrm{FS}^{\mathrm{c}}$ & {$[-00: 27]$} & 0.048 & 0.646 & 0.810 & 0.280 & 6.819 & 0.354 & - & 5.640 \\
\hline
\end{tabular}

by Minakata et al. (2009) where the SAR by Doussin and Monod (2013) fails (see also Table 2).

Branching ratios for the various product channels are determined by scaling the individual rate constants, which have been modulated by the effects of the neighbouring groups, to the overall rate constant. Immediate oxygen addition to alkyl radicals resulting from $\mathrm{H}$ atom abstraction is assumed in GECKO-A and directly produces peroxy radicals. A threshold to omit minor reaction pathways and reduce mechanism size is implemented in GECKO-A where the current protocol uses $3 \%$ as standard. When minor branches are omitted from the generator, the overall rate constant is reduced. No attempts are made to rescale the remaining reaction pathways to the overall rate constant, hence reducing the rate constant when minor reaction pathways are omitted. This could result in a sufficient loss of reactivity if too many minor reaction pathways are omitted, which account for a significant portion of the overall reaction. Therefore, a second parameter is introduced, which ensures that the overall reactivity stays above a defined percentage ( $80 \%$ as standard). In GECKOA, 10 levels of accuracy exist for the thresholds of minor reaction pathways ranging from 0.1 to $25 \%$. If the overall reactivity is reduced below the threshold of $80 \%$ (or any other user input), then the generator automatically switches to the next more accurate threshold level for minor branches until the reactivity stays above the defined threshold or if the highest accuracy of $0.1 \%$ cut-off for minor branches is reached. This is a major improvement to previous CAPRAM versions, where only a few branching ratios with experimental evidence have been implemented in the mechanism. The approach is similar to that by Mouchel-Vallon et al. (2017) in the Cloud Explicit Physico-chemical Scheme (CLEPS 1.0) model, where branching ratios are determined by a SAR, and a reduction was applied where the contribution of each reaction pathway was determined to maintain at least $75 \%$ of the total reactivity. After the reduction, branching ratios were recalculated to maintain the global rate constant (MouchelVallon et al., 2017).

Nitrate radical reactions are estimated with the new advanced Evans-Polanyi-type correlations introduced in Sect. 2.3.3. Evans-Polanyi correlations are designed for gasphase $\mathrm{H}$ atom abstraction reactions only but have proven to work equally well in the aqueous phase (e.g. Hoffmann et al., 2009). However, this results in limitations of the applicability of Evans-Polanyi-type correlations as they are unsuitable for unsaturated compounds and ions. Therefore, estimates with fixed rate constants of $1 \times 10^{7} \mathrm{M}^{-1} \mathrm{~s}^{-1}$ had to be used for these compounds based on the sensitivity studies described in Sect. 3. Moreover, no branching ratios can be derived from these estimates. Therefore, branching ratios from hydroxyl radical reactions were used with the exception of reactions with carboxylates, where electron transfer is assumed to be 
the only process. For unsymmetrical dicarboxylates, equal branching is assumed. There is a need for further development of a prediction method for kinetic and mechanistic data of nitrate radical reactions with organic compounds. However, for more advanced predication methods such as SARs, a more comprehensive experimental database is needed.

Uptake is described with GROMHE. Due to the large uncertainties, which derive from the experimental data used to obtain the relationship, the SAR is only applied to compounds with an $\mathrm{O}: \mathrm{C}$ ratio below 1 . Based on recent findings, higher oxidized compounds are assumed to remain in the aqueous phase until a SAR becomes available. Furthermore, uptake is restricted to species with an HLC between $10^{2}$ and $10^{12} \mathrm{M} \mathrm{atm}^{-1}$. This measure has been taken to reduce the size of the generated mechanism as species below the lower threshold will predominantly exist in the gas phase, and species above the range will almost exclusively remain in the aqueous phase (see also discussions by Mouchel-Vallon et al., 2013). Therefore, reaction schemes for these compounds can be omitted from the corresponding phases with only small errors.

Further parameters needed to describe the phase transfer process are the gas-phase diffusion coefficient $D_{\mathrm{g}}$ and the mass accommodation coefficient $\alpha . D_{\mathrm{g}}$ can be calculated from the molecular diffusion volumes with the FSG method as described by Fuller (1986). For $\alpha$, a fixed value of 0.1 is applied in the new aqueous-phase protocol based on previous CAPRAM estimates and the sensitivity studies presented in Sect. 3.

For the estimation of hydration equilibrium constants of carbonyl compounds, GROMHE can be used as well. Hydrations are important to determine the effective HLCs. Therefore, their prediction is already incorporated in GROMHE and can directly be used for the new GECKO-A protocol. To reduce the size of the generated mechanisms, hydration channels are omitted from equilibria, where the hydration equilibrium constant for a channel is below $5 \%$ of the overall equilibrium constant $k_{\text {hyd }}^{*}$ taking all possible hydration forms into account.

Dissociation of carboxylic acids is estimated using the method by Perrin et al. (1981). No threshold for minor dissociation states is currently used in the standard mode of GECKO-A as the threshold is highly dependent on particle and cloud droplet $\mathrm{pH}$. A varying $\mathrm{pH}$ in the model scenario with non-permanent clouds, as applied in this study, can lead to significant inaccuracies. Furthermore, different dissociation states possess different reactivities. Dissociated carboxylic acids are likely to react by electron transfer reaction (ETR), which are generally faster than $\mathrm{H}$ abstraction reactions (see also Tables S1 and S2 in the ESM). Therefore, with increased reactivities of the products of minor branches, significant turnovers can still be achieved through these reaction pathways. These considerations apply also to hydration processes but are less critical for this equilibrium type with less variation of the reaction rate constants between the different hydration forms. Future versions of the generator should use more advanced determinations of the threshold for minor reaction pathways, which include the consideration of the reactivities of the products in each channel. However, these estimates would depend on typical radical oxidant concentrations and $\mathrm{pH}$ and, hence, would be environment specific.

For highly oxidized polycarbonyl compounds, a monomolecular decay has been implemented in addition to the radical attack if three carbonyl groups are found within four adjacent carbon atoms. Bond breaking occurs always between two carbonyl groups. If three carbonyl groups are directly adjacent to each other, equal branching is assumed leading to a maximum of four fragments. The decay rate is estimated as $0.1 \mathrm{~s}^{-1}$ based on the sensitivity studies in Sect. 3.

For organic nitrates with an $\alpha$-carbonyl group, hydrolysis is assumed in addition to radical attack. The second-order rate constant is assumed equal to peroxyacyl nitrate (PAN) hydrolysis with an estimated second-order rate constant of $k_{2 \text { nd }}=7.6 \times 10^{-6} \mathrm{M}^{-1} \mathrm{~s}^{-1}$ and $E_{\mathrm{A}} / R=6600 \mathrm{~K}$ (Kames and Schurath, 1995). Products formed are a carboxylic acid and dissociated nitric acid.

Due to the experimental difficulties in determining the very fast reaction rate constants of organic radical compounds, measurements are scarce. Therefore, no sophisticated estimation method could be derived for these compounds, and estimates use fixed rate constants and branching ratios based on previous CAPRAM estimates. Organic peroxy radical reactions are based on the mechanism proposed by von Sonntag (1987) and von Sonntag and Schuchmann (1991) with updated mechanistic and kinetic data by Schaefer et al. (2012) and include the reactions and estimated rate constants as given in Table 2. Peroxy radical reactions are included as pseudo first-order reactions based on the methodology of the gas-phase mechanism MCM (Jenkin et al., 1997; Saunders et al., 2003), where a given peroxy radical reacts with the sum of concentrations of all peroxy radicals to account for recombinations and cross-reactions. Several exceptional reaction pathways of certain peroxy radicals have been included in the protocol. Peroxy radicals bearing a hydroxyl group in $\alpha$ position solely decay by $\mathrm{HO}_{2}$ elimination to form a carbonyl compound and a hydroperoxyl radical in a unimolecular decay. Decay rates are estimated at $200 \mathrm{~s}^{-1}$ for one hydroxyl group in $\alpha$ position and at $1000 \mathrm{~s}^{-1}$ for a gem-diol function in $\alpha$ position based on previous CAPRAM estimates (Tilgner and Herrmann, 2010). Recombination and cross-reactions are neglected for this type of peroxy radical due to the considerations given in Sect. S3.5 of the ESM. Peroxy radicals with an adjacent carboxyl or carboxylate group decay by $\mathrm{CO}_{2}$ elimination in addition to the recombination and cross-reactions (see Table 2 for more details on mechanistic and kinetic data). Decay rates are estimated based on the measurements of the acetate peroxy radical $\left(\mathrm{CH}_{2}(\mathrm{OO} \cdot) \mathrm{C}(=\mathrm{O}) \mathrm{O}^{-}\right)$by Schuchmann et al. (1985). 
Alkoxy radicals decay by reaction with $\mathrm{O}_{2}$ or by monomolecular decay and have been implemented in the new protocol with the rate constants determined in Sect. 3.3. Immediate oxygen addition is assumed for any carbon-centred radical fragments, which will directly lead to the formation of peroxy radicals. For acyloxy radicals, a monomolecular decay by $\mathrm{C}-\mathrm{C}$ bond breaking of the acyloxy group is assumed, leading to dissolved carbon dioxide and a peroxy radical as immediate oxygen addition is assumed for the resulting alkyl radical. In contrast to previous CAPRAM estimates, a reduced decay rate of $5 \times 10^{2} \mathrm{~s}^{-1}$ is assumed in the current protocol in accord with the reduced bond breaking rates of alkoxy radicals.

For every auto-generated CAPRAM version, three subversions are created. The standard $\alpha$ version describes the full nitrate radical chemistry. The $\beta$ mechanism includes the full inorganic and organic nitrate radical chemistry of the CAPRAM core mechanism 3.0n but omits any reactions of nitrate radicals with unsaturated organic compounds. This measure significantly reduces the size of the mechanism as organic nitrate formation is suppressed resulting in a significantly reduced number of intermediate species in the oxidation chain of organic compounds. In the most reduced $\gamma$ mechanism, only nitrate radical chemistry of the core mechanism CAPRAM 3.0n is allowed, while nitrate radical chemistry in the auto-generated mechanism part is suppressed. The potential for a significant reduction in mechanism size is far less for the $\gamma$ version than for the $\beta$ version of the mechanism as in the $\gamma$ mechanism the same intermediates are produced as in the corresponding hydroxyl radical reactions. Thus, the mechanism is only reduced by the number of nitrate radical reactions, but no reduction in the number of species is achieved. The latter two sub-versions of the mechanism are meant for modelling in clean environments or for low-NOx chamber simulations.

Two modes of operation exist in CAPRAM-GECKO-A. In the first option, the generator produces mechanisms entirely based on the protocol rules. For every parent compound, a complete mechanism is auto-generated up to the final products $\mathrm{CO}$ and/or $\mathrm{CO}_{2}$. In the second option, the generator produces supplementary modules for a parent mechanism and can be used to extend CAPRAM 3.0n or higher versions. In this mode, the generator stops the auto-generation when compounds of the parent mechanism are reached.

\subsection{Implementation of the protocol in GECKO-A}

The above protocol rules have been implemented as a new module in the existing gas-phase mechanism generator GECKO-A. The gas-phase mechanism generator is described in detail by Aumont et al. (2005). This section details the incorporation of the new aqueous-phase protocol. The workflow of the aqueous-phase module of GECKO-A is schematically shown in Fig. 17. GECKO-A will always be initialized in the gas phase as most organic compounds are emitted as trace gases. However, it is possible to extract just the aqueous-phase mechanism from GECKO-A and couple it to further mechanisms as done in this study.

To generate oxidation schemes with GECKO-A, any number of "primary gas-phase species", for which an oxidation mechanism is desired, can be added to the "primary stack". The mechanism generator will start with the first species in this stack and generate all possible gas-phase reactions as described by Aumont et al. (2005). In a second step, HLCs will be generated by the GROMHE subroutine and phase transfer will be described in the generated mechanisms if the HLC is above a given threshold. Otherwise, the assumption is made that the compound is insoluble and exclusively available in the gas phase. Therefore, uptake is suppressed to reduce the aqueous-phase mechanism size.

In the next step, the protocol rules described in Sect. 4.1 will be applied (see also Fig. 17). This includes checks for radical functions and any other functional groups in the molecule and the application of the appropriate rules from the protocol as schematically depicted in Fig. 17. The generator determines any mechanistic, kinetic, and thermodynamic data for the given compound. Furthermore, it checks whether reaction products have previously been treated. New intermediate species will be added to an "aqueous stack" for later treatment. Before the data are written into a mechanism, the solubility of the compound is checked again. If the HLC is below a pre-defined threshold, release to the gas phase is treated. The compound will be checked for its treatment in the gas phase. Untreated species will be added to a gaseous stack for later treatment in the gas-phase mechanism. If a compound exceeds the HLC limit, exclusive existence in the aqueous phase is assumed to reduce the size of the gas-phase mechanism.

In a last check, GECKO-A consults an experimental database. Any available experimental kinetic or thermodynamic data are preferred over theoretically predicted data. All reactions for the given compound are written into a mechanism as determined by GECKO-A. The generator then returns to the aqueous stack for untreated species and generates reactions for these compounds as well. Thus, the aqueous stack will be filled by new intermediate species from the generated reactions. After the treatment of a species it will be marked to avoid double treatment of species generated in several reactions. GECKO-A proceeds with all unmarked species in the aqueous stack until every species in the stack is marked and the products of a current reaction are only species already treated previously or the end products $\mathrm{CO}$ and/or $\mathrm{CO}_{2}$. In this case, the generator returns to the gasphase stack and continues to generate gas-phase reactions for any unmarked species in this stack. The gas and aqueousphase mechanism will be generated alternately in this way until a complete multiphase mechanism is generated for the species of the primary stack and all intermediate products.

After the generation of a complete multiphase oxidation scheme for a species of the primary stack, the generator re- 


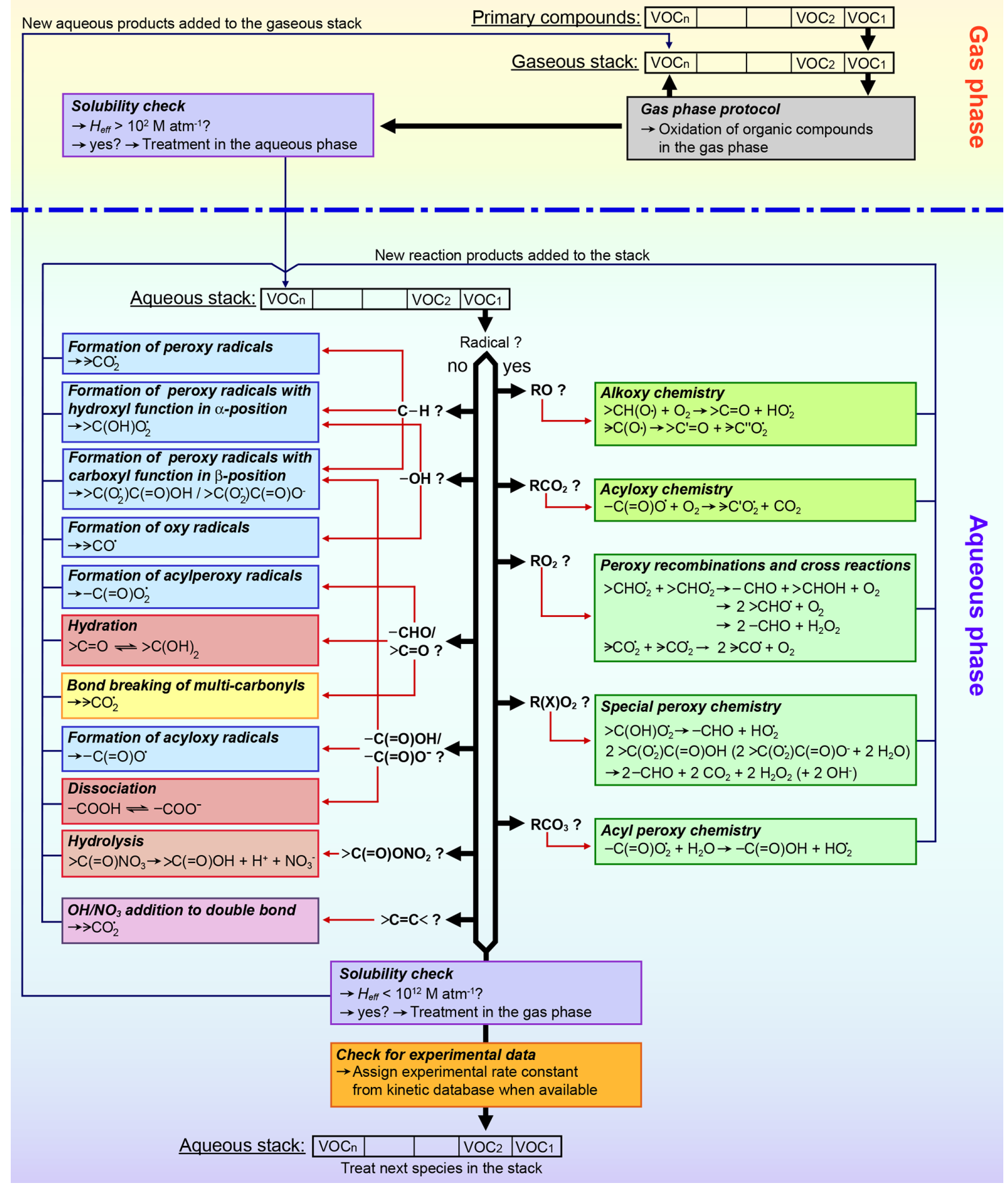

Figure 17. Workflow of the generator GECKO-A with the processes implemented in the aqueous-phase protocol. Blue boxes represent $\mathrm{H}$ abstraction reactions, the pink box represents radical addition to double bonds of unsaturated organic compounds, red boxes represent equilibria, the yellow box represents $\mathrm{C}-\mathrm{C}$ bond breaking of poly-carbonyls, the light brown box represents the hydrolysis of carbonyl nitrates, and green boxes represent radical chemistry (different shades differentiate between oxy and peroxy radical chemistry).

turns to this stack to treat the next species in it. The reaction mechanism for this compound will complement the mechanism of the previous species of this stack. Therefore, species of the gaseous and aqueous stack will not be emptied until the complete treatment of the primary stack. Instead, they will be used as markers to indicate previous treatment in the generator and avoid double reactions. GECKO-A finishes when all species of the primary, gas, and 'aqueous stacks are marked as treated and a complete multiphase reaction scheme has been generated for these species. 


\section{Validation of the CAPRAM-GECKO-A protocol against aerosol chamber experiments}

\subsection{Chamber experiments}

The oxidation of 1,3,5-trimethyl benzene (TMB) was conducted in the aerosol chamber LEAK (Leipziger Aerosolkammer). A detailed description of LEAK can be found elsewhere (Mutzel et al., 2016 and references therein). The experiments were conducted in the presence of sodium sulfate seed particles mixed with $30 \%$ sodium peroxide. The photolysis of ozone with UV-C light $(\lambda=254 \mathrm{~nm})$ served as gasphase $\mathrm{OH}$ radical source (see Reactions 9 and 10). Ozone was generated by UV irradiation of $\mathrm{O}_{2}$ using a flow rate of $3 \mathrm{~L} \mathrm{~min}^{-1}$.

$\mathrm{O}_{3}+\mathrm{h} v \longrightarrow \mathrm{O}_{2}+\mathrm{O}\left({ }^{1} \mathrm{D}\right)$

$\mathrm{O}\left({ }^{1} \mathrm{D}\right)+\mathrm{H}_{2} \mathrm{O} \longrightarrow 2 \mathrm{OH}$

Furthermore, sodium peroxide was used as $\mathrm{OH}$ radical in situ source in the particle phase via the following reaction sequence.

$\mathrm{Na}_{2} \mathrm{O}_{2}+2 \mathrm{H}_{2} \mathrm{O} \longrightarrow 2 \mathrm{NaOH}+\mathrm{H}_{2} \mathrm{O}_{2}$

$\mathrm{H}_{2} \mathrm{O}_{2}+h v \longrightarrow 2 \cdot \mathrm{OH}$

TMB $(\approx 85.5 \mathrm{ppb})$ was injected into LEAK with a Microliter syringe $(50 \mu \mathrm{L}$ gastight springe Hamilton-Bonaduz, Schweiz). The oxidation of TMB was conducted at a relative humidity of $75 \%$ and at room temperature. The consumption of the precursor compound $(\triangle \mathrm{HC})$ was monitored with a proton-transfer-reaction mass spectrometer (PTR-MS) over a reaction time of $210 \mathrm{~min}$. The volume size distribution of the seed particles was measured with a scanning mobility particle sizer (SMPS). An average density of $1 \mathrm{~g} \mathrm{~cm}^{-3}$ was used to calculate the produced organic particle mass $(\Delta \mathrm{M})$. The particle phase was sampled after the experiments, and 1.8 and $0.6 \mathrm{~m}^{3}$ of the chamber volume was collected on a PTFE filter (borosilicate glass fibre filter coated with fluorocarbon; $47 \mathrm{~mm}$ in diameter; PALLFLEX T60A20, PALL, NY, USA), which was connected to a denuder (URG-200030B5, URG Corporation, Chapel Hill, NC, USA; Kahnt et al., 2011) to avoid gas-phase artefacts. The particle-phase products were also collected with a condensation growth and impaction system (C-GIS, Sierau et al., 2003) with a time resolution of $15 \mathrm{~min}$. Particle mass was monitored by an aerosol mass spectrometer (high-resolution time-of-flight aerosol mass spectrometer; HR-ToF-AMS).

\subsection{Model setup}

For the gas-phase description of the chemistry, the MCMv3.2 was used (http://mcm.leeds.ac.uk/MCMv3.2/, last access: 5 October 2015; Jenkin et al., 2003; Saunders et al., 2003). GECKO-A was initialized only with methylglyoxal as both the chamber and model results (see results in Sect. 5.3) did

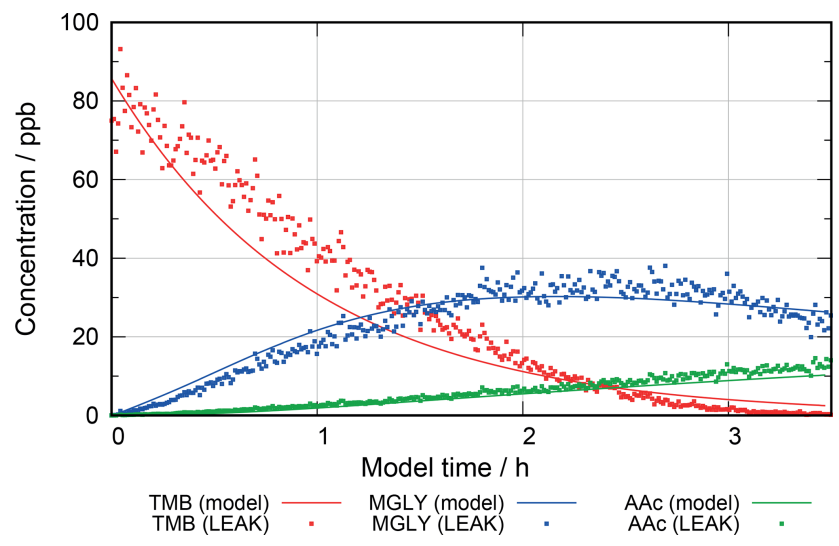

Figure 18. Comparison of measured (squares) and modelled (lines) gas-phase volume mixing ratios for 1,3,5-trimethyl benzene (TMB, red), methylglyoxal (MGLY, blue), and acetic acid (AAc, green) in the model scenario $\mathrm{RXN}_{0.4}$.

not show significant concentrations of any other intermediate compound in the oxidation chain between TMB and methylglyoxal. The final version of GECKO-A, as described above, was used to generate the aqueous-phase oxidation scheme including phase transfers (with HLCs set to $1 \times 10^{9} \mathrm{M} \mathrm{atm}^{-1}$ for species with an $\mathrm{O}: \mathrm{C}$ ratio greater than or equal to 1 ). The generated scheme includes important aqueous-phase compounds such as oxalate, pyruvate, acetate, formate, formaldehyde, or several di- and polycarbonyls. The total aqueousphase scheme comprises 264 species, 596 reactions, and 25 phase transfers. The mechanism is supplied as SPACCIM input file in the ESM.

Despite very low gas-phase concentrations, chemical turnovers can be substantial for some of the larger TMB oxidation products. Furthermore, some of the larger intermediates are already very oxidized with high $\mathrm{O}: \mathrm{C}$ ratios, and the aqueous-phase might be a potent sink for them. Therefore, several sensitivity studies have been conducted where uptake into the aqueous phase was considered for 20 additional intermediates. As some of the intermediates were still very large with carbon numbers up to nine, an explicit description model with the currently very detailed protocol is beyond the capabilities of the current box with its detailed microphysical scheme. Therefore, the generic model species WSOC was used as a reaction product for those intermediates in the sensitivity run "UPT".

As the majority of those intermediates bear double bonds, and carbonyl and/or carboxyl functional groups, reactive uptake is possible due to the fast kinetics (addition reactions, hydrolysis, and dissociations) of those compound classes. Therefore, another sensitivity run "RXN" was performed, where the initial aqueous-phase reactions were described explicitly following the generator protocol rules. Reaction products were described only, if they were part of the explicit methylglyoxal oxidation scheme, otherwise WSOCs were 


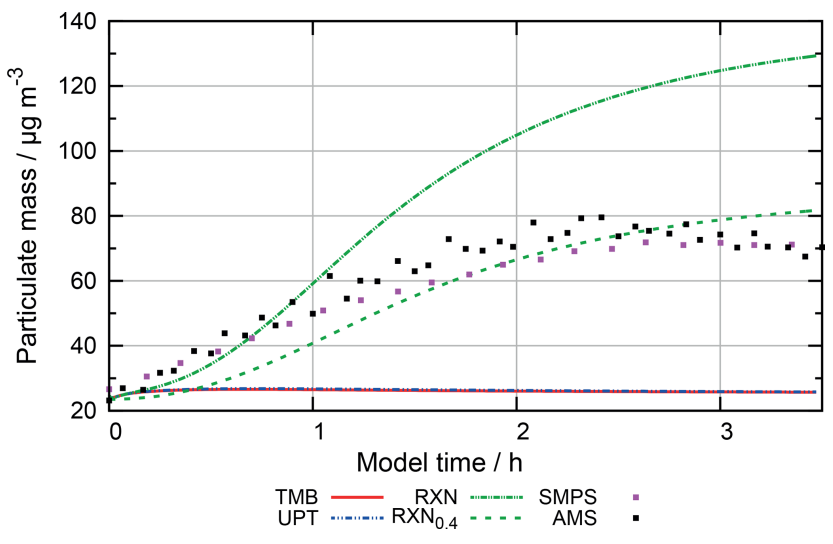

Figure 19. Comparison of dry particle mass in the various sensitivity studies with SMPS and AMS measurements.

used as reaction products. In a last sensitivity run, WSOC yields in the additional reaction scheme of the "RXN" run were reduced to 0.4 to match the model predictions of the particle mass with the AMS (aerosol mass spectrometer) and SMPS measurements. The additional species and reactions of the sensitivity runs "UPT", "RXN", and "RXN 0.4 " alongside the kinetic and thermodynamic data used can be found in Table S15 in the ESM.

Model runs were performed with the box model SPACCIM (Wolke et al., 2005) with a detailed description of microphysical and chemical processes. The model was initialized with $2.14 \times 10^{12}$ molecules $\mathrm{cm}^{-3}$ of "TMB", $3.11 \times 10^{10}$ molecules $\mathrm{cm}^{-3}$ of ozone and traces of NO and $\mathrm{NO}_{2}$ to account for any background chemistry (1.5 and $2.0 \times 10^{10}$ molecules $\mathrm{cm}^{-3}$, respectively). The aqueousphase was initialized with sodium sulfate particles and $30 \%$ sodium peroxide. $\mathrm{OH}$ concentrations were held constant in both phases at a gas-phase concentration level of $5 \times 10^{6}$ molecules $\mathrm{cm}^{-3}$ and an aqueous concentration of $10^{-11} \mathrm{M}$. This was necessary as the current model version is designed specifically for atmospheric applications with a solar radiation spectrum under tropospheric conditions. Thus, it was not possible to simulate a constant light source from non-solar irradiation. The same microphysical conditions as in the chamber experiments were used with constant relative humidity of $75 \%$ and a constant temperature of $298 \mathrm{~K}$.

\subsection{Comparison of chamber and model results}

Figure 18 shows the gas-phase concentration time profiles of TMB and its major oxidation products methylglyoxal and acetic acid as monitored by PTR-MS (squares) or simulated in the model run $\mathrm{RXN}_{0.4}$. It should be noted that concentrations for methylglyoxal and acetic acid had to be scaled by a factor of 1.35 and 0.14 , respectively, which can at least in part be explained by different protonation efficiencies for different molecules in the PTR-MS. This leads to inaccuracies in the PTR-MS measurements, which justify a re-scaling.
Another, yet more unlikely, source of error would be another overlying compound with the same mass-to-charge ratio, which modifies the PTR-MS measurements for the respective $m / z$.

With the rescaled concentrations, however, the MCMv3.2 is able to explain the measured concentrations well. The general degradation of TMB and the subsequent formation of methylglyoxal and acetic acid are well captured by the model. The concentration-time profile of TMB shows a slightly steeper curve in the model results with slightly stronger degradation rates in the first $1.5 \mathrm{~h}$ and slower degradation rates thereafter. Up to $15 \mathrm{ppb}$ less TMB are observed in the model compared to the experiment in the first hour. Consequently, methylglyoxal production is slightly over-predicted in the first $1.5 \mathrm{~h}$, leading to $\sim 2-3 \mathrm{ppb}$ more methylglyoxal in the model compared to the measurements, and the magnitude of the decay of methylglyoxal towards the end of the experiment is smaller in the model than in the measured concentration-time profile. At the end of the experiment, the model overpredicts the experimental results by about $3 \mathrm{ppb}$. Agreement of acetic acid concentrations between model and experiment is very good with slightly lower modelled concentrations towards the end of the experiment (2-3 ppb).

Possible explanations for the discrepancies between measured and modelled concentrations in Fig. 18 are uncertainties in the mechanistic and kinetic data either directly in the gas phase or indirectly in the aqueous phase, influencing gasphase concentrations through reactive uptake. Even though only TMB, methylglyoxal, and acetic acid have been measured in significant concentrations, it is likely that uptake of further intermediates in the oxidation chain of TMB to methylglyoxal influence the concentration profiles. Despite minor concentrations, large uptake fluxes into aerosol particle are modelled for the highly oxidized intermediates. With uptake processes having one of the most uncertain estimates in the CAPRAM-GECKO-A protocol (see Sect. 3), they are another likely source of the observed discrepancies between modelled and measured concentrations.

In the aqueous phase, methylglyoxal chemistry alone fails to predict an increase in particulate matter (PM) by approximately $50 \mathrm{\mu g} \mathrm{m}^{-3}$ as seen by the SMPS and AMS (see Fig. 19). Particle growth is negligible in the model run TMB. Therefore, more sensitivity runs have been performed as introduced in Sect. 5.2, where uptake of further precursors of methylglyoxal and their initial reaction steps in the aqueous phase are considered. The influence of uptake alone in the sensitivity run UPT is negligible and the concentration-time profile of the overall dry particle mass is indistinguishable from the base run TMB. Aqueous-phase chemistry has a big influence on particle growth. In the simulation RXN, besides uptake, initial reactions are also considered assuming $100 \%$ water-soluble organic compounds (WSOCs) as the reaction product, and model predictions of PM at the end of the experiment are at $\sim 130 \mu \mathrm{g} \mathrm{m}^{-3}$ about twice as high as those 

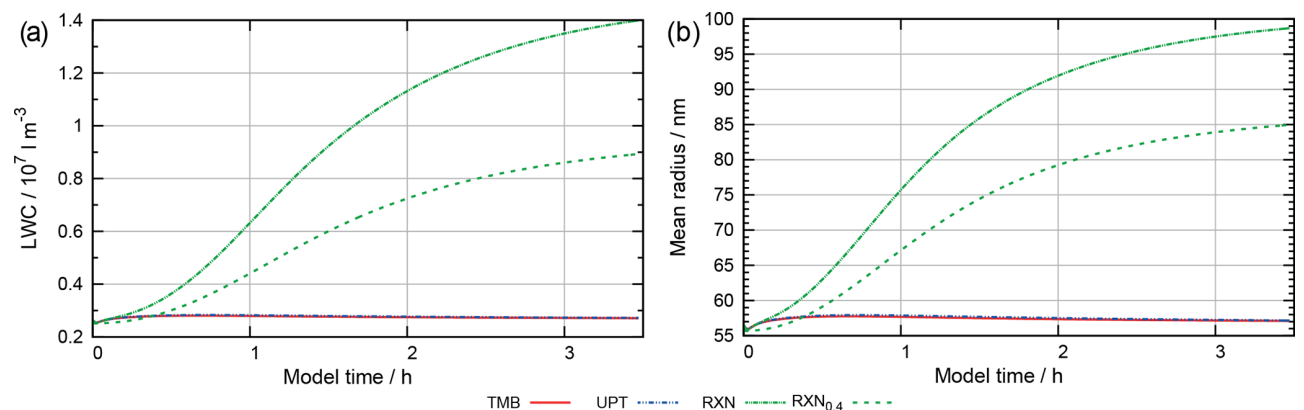

Figure 20. Profiles of the liquid water content (LWC, panel a) and mean particle radius (b) for the various model runs.

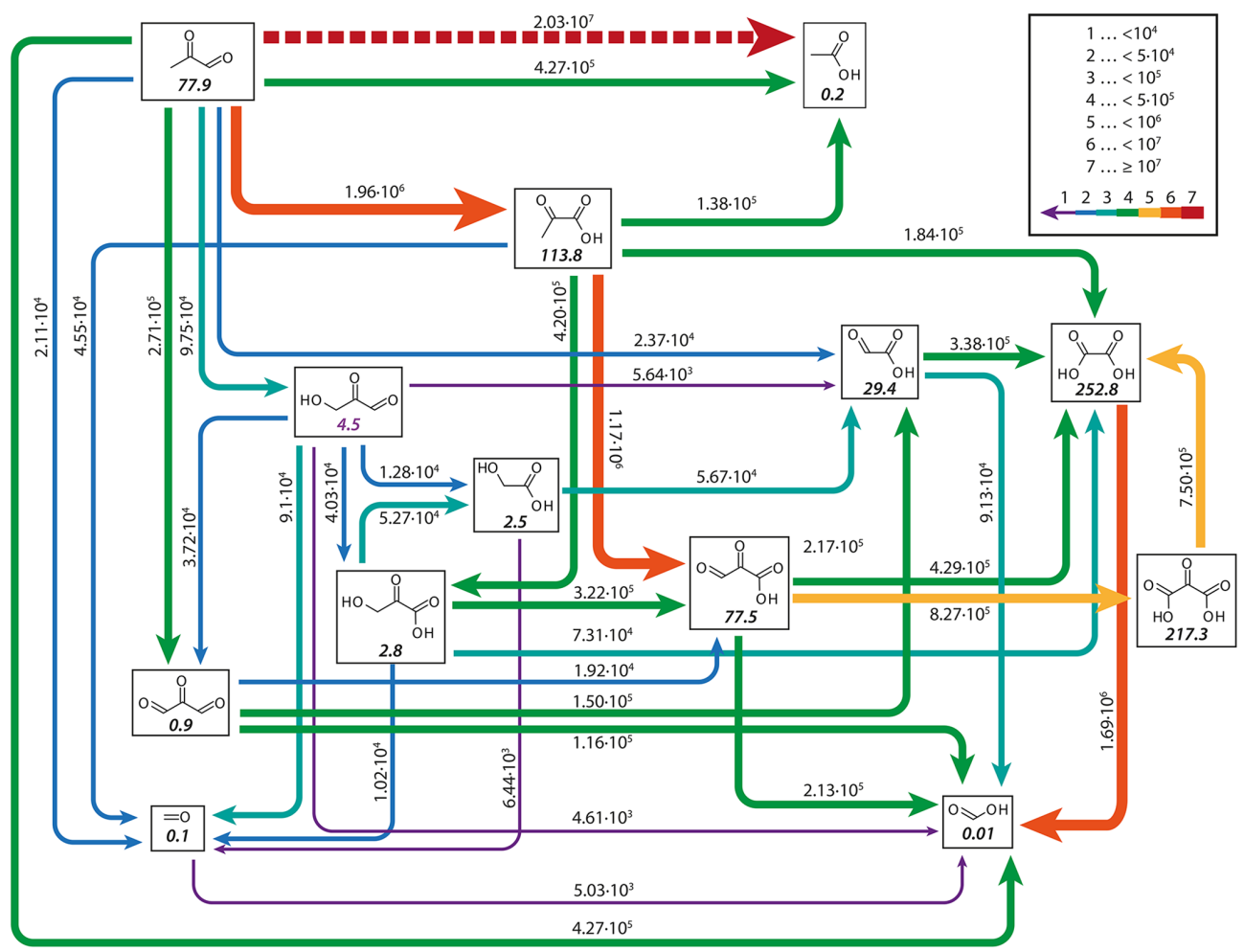

Figure 21. Chemical fluxes in the TMB oxidation experiments modelled in the scenario " $\mathrm{RXN} \mathrm{N}_{0}$ " averaged over the whole $3.5 \mathrm{~h}$ of the experiment. Numbers above the arrows correspond to fluxes in molecules in cubic centimetres per second $\left(\mathrm{cm}^{-3} \mathrm{~s}^{-1}\right)$, and bold numbers in the species boxes are concentrations in nanograms per cubic metre $\left(\mathrm{ng} \mathrm{m}^{-3}\right)$ at the end of the experiment.

seen by the SMPS or AMS. Reasonable agreement between modelled and measured concentrations is reached only when the reaction product WSOCs is reduced by an artificial stoichiometric index of 0.4. This rescaling also has an impact on gas-phase concentrations, decreasing the concentrations of methylglyoxal and, consequently, acetic acid. To match the concentrations in the best scenario $\mathrm{RXN}_{0.4}$ to experimental results, scaling factors for methylglyoxal in the gas phase were improved from 2.5 to 1.35 , while acetic acid decreased from 0.23 to 0.14 . The comparison of all gas-phase concentrations of methylglyoxal and acetic acid can be found in Fig. S18 in the ESM.
Hence, large oxidation products are needed to explain the particle growth seen in the experiment, despite their negligible concentrations in the gas phase. The scaling factor for WSOCs of 0.4 in the scenario $\mathrm{RXN}_{0.4}$ could be interpreted in a way that subsequent aqueous-phase chemistry produces a mixture of soluble, semi-volatile, and volatile products of which $40 \%$ will remain in the aqueous phase, and $60 \%$ are released back to the gas phase. This interpretation might also be used as a justification for the newly introduced degradation rates for alkoxy radicals. While previous CAPRAM mechanisms used fragmentation as the predominant degradation pathway, products are shifted towards carbonyl formation with a ratio of $73 \%$ carbonyl formation and $27 \%$ frag- 
mentation under the conditions of the experiment. As carbonyl formation decreases volatility in contrast to fragmentation, the new product distribution is in better agreement with the above results from the sensitivity runs $\mathrm{RXN}$ and $\mathrm{RXN}_{0.4}$, assuming that not all carbonyl compounds will remain completely in the aqueous phase.

However, even in the final rescaled sensitivity run $\mathrm{RXN}_{0.4}$ some discrepancies can be observed. The increase in PM is delayed by about $20 \mathrm{~min}$ in the model run. Furthermore, the model does not predict a slight decrease in PM towards the end of the model run as seen by the SMPS and AMS. The delayed increase is somewhat surprising and its reason remains unclear as an increased degradation of TMB was observed in the first $1.5 \mathrm{~h}$ of the experiment in Fig. 18, which would imply an increased particle formation in the model runs compared to the measurements. However, even in the run RXN, where $100 \%$ WSOCs formation is assumed, PM formation is slightly lower in the first 40 min of the experiment than observed by the AMS and SMPS. It should also be mentioned that no wall losses of particles and oxidized organic compounds were implemented in the model as SPACCIM mainly targets atmospheric applications. Disregarding of chamber background chemistry is another source of discrepancies. Moreover, currently no non-radical chemistry, such as accretion reactions, is implemented in the CAPRAM-GECKO-A protocol, albeit no oligomers were observed in the experimental particle analysis.

Further investigations of the microphysical parameters liquid water content (LWC) and mean particle radius in Fig. 20 show more interesting aspects of the influence of aqueous chemistry on particle growth. It seems that particle chemistry is necessary to initiate particle growth. Without aqueous-phase chemistry of the larger intermediates, no particle growth is observed in the run TMB. Uptake of these compounds alone is not sufficient to overcome the curvature effect of the relatively small seed particles with a mean radius of $55 \mathrm{~nm}$. However, if chemistry is treated in the runs RXN and $\mathrm{RXN}_{0.4}$, particle radii and, hence, LWC increase rapidly. The chemistry is needed as a trigger for the initial growth. With growing radii and decreasing curvature effect, LWC increases and uptake processes have an increased importance for the particle composition.

The increased uptake of organic compounds and, hence, increased carboxylic acid formation in the scenarios RXN and $\mathrm{RXN}_{0.4}$ causes a decrease in $\mathrm{pH}$ by about 1 unit. The decrease from near neutral concentration is strongest in the first minutes of the experiments and levels off after 1 to $2 \mathrm{~h}$, as can be seen in Fig. S16 of the ESM.

Attempts were made to identify concentrations of the expected aqueous-phase products by offline analysis of C-GIS and PTFE filter samples. However, concentrations were close to the detection limit or within the noise of the expected background chemistry as can be seen in Table 3. In this table, the first sample represents background concentrations prior to the injection of $1,3,5-\mathrm{TMB}$ and ozone. Moreover, large differences are seen in the concentrations for some of the compounds determined from samples using the different sampling techniques. For example, oxalate concentrations from filter samples were with $6.8 \mu \mathrm{g} \mathrm{m}^{-3}$ almost 10 times as high as determined from the C-GIS samples $\left(0.7 \mu \mathrm{g} \mathrm{m}^{-3}\right)$. Therefore, a direct comparison of concentrations of individual species of experimental and modelled results is difficult.

It should be mentioned that large amounts of malic acid were detected in the gas and the aqueous phases throughout the experiment as shown for the gas phase in Fig. S17 in the ESM and for the aqueous phase in Table 3. However, there is currently no information about formation pathways of malic acid from TMB oxidation and concentrations are attributed to background chemistry. Consequently, the MCMv3.2 does not predict the formation of malic acid in any of the sensitivity runs.

For a thorough analysis, modelled chemical fluxes from the explicit scheme in scenario $\mathrm{RXN}_{0.4}$ have been analysed, and averages over the whole $3.5 \mathrm{~h}$ of the experiments are shown in Fig. 21. In addition, final aqueous-phase concentrations after $3.5 \mathrm{~h}$ are shown for each species. Figure 21 shows that less than $3 \%$ of methylglyoxal is oxidized to acetic acid in the aqueous phase, and gas-phase oxidation is the main sink. In the aqueous phase, $75 \%$ of the acetic acid is formed directly from methylglyoxal while $25 \%$ is formed with pyruvic acid as intermediate, which is the major first generation aqueous-phase oxidation product. Oxidation of pyruvate leads mainly to oxo-pyruvic acid, which is subsequently degraded to mesoxalic acid and oxalate in the model and finally to formate. The formation of these highly oxidized dicarboxylic acids is a direct consequence of the revision of the radical protocol in the CAPRAM-GECKO-A mechanism generation process. The revised monomolecular decay of alkoxy radicals after the hydroxyl radical attack of pyruvic acid leads to an increased formation of oxo-pyruvic acid in favour of fragmentation. Previous CAPRAM mechanisms would have favoured the formation of more acetic acid. Thus, the new protocol favours particle growth, as mesoxalic and oxalic acid are far less volatile than acetic acid. The modelled concentrations in Fig. 21 in nanograms per cubic metre $\left(\mathrm{ng} \mathrm{m}^{-3}\right)$ are lower than those seen in the filter and C-GIS samples (see Table 3) but are generally within the experimental error. An exception is methylglyoxal with concentration modelled somewhat higher $\left(78 \mathrm{ng} \mathrm{m}^{-3}\right)$ compared to measurements $\left(48 \mathrm{ng} \mathrm{m}^{-3}\right)$.

Despite the difficulties to conduct experiments with sufficient bulk aqueous-phase chemistry, the plausibility of the new CAPRAM-GECKO-A protocol could be proven, while further validation from model simulations with larger mechanisms under real tropospheric conditions is needed. These studies will be presented in a companion paper. 


\section{Conclusions}

A new protocol for mechanism auto-generation within the expert system GECKO-A has been developed and tested. The protocol was developed to supplement the mechanism generation process of the aqueous-phase benchmark mechanism CAPRAM.

Following an extensive evaluation of existing kinetic data and prediction methods for aqueous-phase mechanistic, thermodynamic, and kinetic data, Evans-Polanyi-type correlations have been further improved. The advanced correlations consider all bonds in a molecule connected to an $\mathrm{H}$ atom rather than only the weakest bonds. This way, minor channels are considered in the correlation, which decreases biases especially for compounds, where measurements are typically scarce. Interesting effects were observed, especially for hydroxyl radical reactions. While previously BDE and second-order rate constants were anti-correlated due to an increased reactivity of weaker bonds, the new correlation shows a positive relationship due to increased side attacks on larger molecules, which have a larger accumulated bond dissociation enthalpy ( $\left.\sum \mathrm{BDE}\right)$. Moreover, for hydroxyl radicals quadratic relationships were seen, while nitrate radical reactions can still be described with linear relationships. The most likely explanation for this behaviour is that for larger organic compounds the diffusion limit of the reaction with $\mathrm{OH}$ is reached and the levelling off of the rate constants can best be described with quadratic relationships, while nitrate radical reactions are well below a diffusion-controlled range where linear relationships are still applicable.

The evaluation process led to a new CAPRAM-GECKOA protocol for mechanism auto-generation where hydroxyl radical reactions are estimated with structure-activity relationships by Doussin and Monod (2013) supplemented by SARs from Minakata et al. (2009). The advanced EvansPolanyi-type correlations were used to describe nitrate radical reactions with organic compounds and estimates with fixed rate constants, and branching ratios were used to predict reactions of organic radicals. Hydrations are estimated with the SAR GROMHE and dissociations using Taft parameters with the method by Perrin et al. (1981). Uptake is described with the Schwartz approach (Schwartz, 1986), using GROMHE for the estimation of HLCs, and the FSG method (Fuller, 1986) for the estimation of gas-phase diffusion coefficients and constant mass accommodation coefficients $\alpha=0.1$.

Rigorous testing in sensitivity studies led to further improvements of the protocol, such as a monomolecular decay channel of polycarbonyl compounds with at least three carbonyl groups within four carbon-containing groups. Furthermore, the largest uncertainties in current estimation methods were identified and led to further improvements of the protocol. These were found for (i) uptake processes and (ii) radical reactions. While uncertainties in uptake processes are generally high, recent research seems to additionally indi- cate that GROMHE is underestimating uptake. Therefore, the current protocol refrains from using GROMHE for very polar molecules with an $\mathrm{O}: \mathrm{C}$ ratio of 1 or greater. Instead, an HLC of $1 \times 10^{9} \mathrm{M} \mathrm{atm}^{-1}$ is used. Moreover, radical reactions are based on only a very few measurements for peroxy radicals and recent estimates from previous CAPRAM studies for alkoxy radicals. The latter radicals are either fragmenting or reacting with dissolved oxygen to form carbonyl compounds. The ratio has a large impact on particle growth as carbonyl formation leads to less volatile products than fragmentation. Therefore, the ratio was refined to best resemble experimental findings. Further sensitivity studies focused on organic nitrate formation in the aqueous phase as alkyl nitrate concentrations are at the upper end of observations or overestimated in the current sensitivity studies.

A TMB oxidation experiment was conducted at the aerosol chamber LEAK, which could prove the plausibility of the current protocol for automated mechanism construction. Modelled gas-phase concentrations of TMB, methylglyoxal, and acetic acid are in good agreement with the PTR-MS measurements. Sensitivity studies have shown that the uptake and aqueous-phase chemistry of larger methylglyoxal precursor species are needed to accurately describe particle growth. However, the currently very explicit protocol would lead to very large and detailed mechanisms for the considered compounds with up to nine carbon atoms, and tests are infeasible for the current version of the box model SPACCIM.

Therefore, more model studies with more comprehensive mechanisms under real atmospheric conditions are needed for the validation of the protocol. The studies are part of a companion paper with further CAPRAM mechanism development. 


\section{Appendix A}

Table A1. A list of abbreviations.

\begin{tabular}{|c|c|}
\hline \multicolumn{2}{|c|}{ A.1 General abbreviations } \\
\hline $\mathrm{BDE}$ & bond dissociation enthalpy \\
\hline ESM & electronic supplementary material \\
\hline ETR & electron transfer reaction \\
\hline HLC & Henry's law coefficient \\
\hline SAR & structure-activity \\
\hline PAN & Peroxyacyl nitrate \\
\hline DCAs & Dicarboxylic acids \\
\hline MCAs & Monocarboxylic acids \\
\hline FSG & Fuller-Schettler-Giddings method \\
\hline \multicolumn{2}{|c|}{ A.2 Scenarios names } \\
\hline woBB & no bond breaking, oxidation by $\mathrm{OH}$ radicals \\
\hline $\mathrm{BBe}-2+\mathrm{OH}$ & bond breaking with $10^{-2} \mathrm{~s}^{-1}$, additional oxidation by $\mathrm{OH}$ radicals \\
\hline BBe-2woOH & bond breaking with $10^{-2} \mathrm{~s}^{-1}$, no additional oxidation by $\mathrm{OH}$ radicals \\
\hline $\mathrm{BBe}-1+\mathrm{OH}$ & bond breaking with $10^{-1} \mathrm{~s}^{-1}$, additional oxidation by $\mathrm{OH}$ radicals \\
\hline BBe-1woOH & bond breaking with $10^{-1} \mathrm{~s}^{-1}$, no additional oxidation by $\mathrm{OH}$ radicals \\
\hline $\mathrm{BBe} 0+\mathrm{OH}$ & bond breaking with $1 \mathrm{~s}^{-1}$, additional oxidation by $\mathrm{OH}$ radicals \\
\hline BBe0woOH & bond breaking with $1 \mathrm{~s}^{-1}$, no additional oxidation by $\mathrm{OH}$ radicals \\
\hline \multicolumn{2}{|c|}{ Sensitivity studies on processing of organic mass fraction } \\
\hline orig & $\begin{array}{l}\text { base scenario, where chemistry of stable compounds in CAPRAM 3.0n has been replaced by a } \\
\text { preliminary CAPRAM-GECKO-A protocol }\end{array}$ \\
\hline WSOC & base with additional chemistry of residual OM (WSOC or WISOC or HULIS species) \\
\hline Phot & scenario WSOC with additional upper limit estimates for organic nitrate photolysis \\
\hline NO3add & $\begin{array}{l}\text { scenario WSOC with updated reaction rate constants of } \mathrm{NO}_{3} \text { radicals with unsaturated organic } \\
\text { compounds }\end{array}$ \\
\hline $\mathrm{O}: \mathrm{C}=1$ & $\begin{array}{l}\text { scenario NO3add with HLCs set to } 10^{9} \mathrm{M} \mathrm{atm}^{-1} \text { for organic compounds with an } \mathrm{O}: \mathrm{C} \text { ratio of } \\
1 \text { or larger }\end{array}$ \\
\hline \multicolumn{2}{|l|}{ Chamber experiments } \\
\hline TMB & $\begin{array}{l}\text { base run for LEAK TMB oxidation experiment using ozone photolysis to generate } \mathrm{OH} \text { radical } \\
\text { oxidants }\end{array}$ \\
\hline UPT & $\begin{array}{l}\text { base run with additional uptake processes for larger organic compounds but no additional } \\
\text { aqueous-phase chemistry }\end{array}$ \\
\hline RXN & $\begin{array}{l}\text { scenario UPT with the first reaction step protocol in the mechanism described by the new } \\
\text { CAPRAM-GECKO-A protocol leading to WSOC for products not defined in the explicit chem- } \\
\text { ical scheme }\end{array}$ \\
\hline $\mathrm{RXN}_{0.4}$ & same as scenario RXN but with WSOC products scaled by a factor of 0.4 \\
\hline \multicolumn{2}{|c|}{ A.3 Model and mechanism names } \\
\hline CAPRAM & $\begin{array}{l}\text { Chemical Aqueous Phase Radical Mechanism (http://projects.tropos.de/capram/, last access: } 3 \\
\text { December 2018) }\end{array}$ \\
\hline GECKO-A & $\begin{array}{l}\text { Generator for Explicit Chemistry and Kinetics of Organics in the Atmosphere (http://geckoa. } \\
\text { lisa.u-pec.fr/index.php, last access: } 3 \text { December 2018) }\end{array}$ \\
\hline GROMHE & Group contribution method for Henry's law estimate (SAR by Raventos-Duran et al., 2010) \\
\hline $\mathrm{MCM}$ & Master Chemical Mechanism (http://mcm.leeds.ac.uk/, last access: 5 October 2015) \\
\hline SPACCIM & SPectral Aerosol Cloud Chemistry Interaction Model \\
\hline \multicolumn{2}{|c|}{ A.4 Chamber and instrumentation abbreviations } \\
\hline AMS & aerosol mass spectrometer \\
\hline C-GIS & condensation growth and impaction system \\
\hline LEAK & Leipziger Aerosolkammer \\
\hline PTFE & polytetrafluoroethylene \\
\hline PTR-MS & proton-transfer-reaction mass spectrometer \\
\hline SMPS & scanning mobility particle sizer \\
\hline \multicolumn{2}{|c|}{ A.5 Organic compound names } \\
\hline HULISs or HULISs $^{-}$ & humic-like substances (fully protonated or deprotonated) \\
\hline $\mathrm{OM}$ & organic matter \\
\hline TMB & 1,3,5-trimethylbenzene \\
\hline WISOCs & water-insoluble organic compounds \\
\hline WSOCs & water-soluble organic compounds \\
\hline 2O3H-SA & 2-oxo-3-hydroxy-succinaldehyde \\
\hline
\end{tabular}


Data availability. The CAPRAM mechanism code is provided in the Appendix. Further datasets, including chamber data and model simulation data used in this study can be accessed by contacting the corresponding author (H. Herrmann: herrmann@tropos.de).

Supplement. The supplement related to this article is available online at: https://doi.org/10.5194/acp-19-9209-2019-supplement.

Author contributions. PB, CMV, AT, BA, and HH developed the protocol for mechanism auto-generation. PB, CMV, AT, RW, BA, and $\mathrm{HH}$ designed the model experiments. $\mathrm{PB}, \mathrm{CMV}$, and BA did model development on GECKO-A. PB and RW did model development on SPACCIM. PB, AT, AM, OB, MR, LP, DvP, and HH designed the aerosol chamber experiments. MR, AM, OB, LP, and DvP performed the aerosol chamber experiments and analysed the data. $\mathrm{PB}, \mathrm{AT}$, and $\mathrm{HH}$ analysed model results. $\mathrm{PB}, \mathrm{AT}$, and $\mathrm{HH}$ wrote the paper.

Competing interests. The authors declare that they have no conflict of interest.

Special issue statement. This article is part of the special issue "Simulation chambers as tools in atmospheric research (AMT/ACP/GMD inter-journal SI)". It is not associated with a conference.

Acknowledgements. This work was performed within the project ATMOCHEM funded by the German Research Foundation (Deutsche Forschungsgemeinschaft, DFG) under the project number BO 1714/3-1. This work has received funding from the European Union's Horizon 2020 research and innovation programme through the EUROCHAMP-2020 Infrastructure Activity under grant agreement no 730997.

Financial support. This research has been supported by the Deutsche Forschungsgemeinschaft (grant no. BO 1714/3-1) and the European Commission (grant no. EUROCHAMP-2020 (730997)).

Review statement. This paper was edited by Andreas Hofzumahaus and reviewed by Andrea Chlebikova and one anonymous referee.

\section{References}

Alfassi, Z. B., Padmaja, S., Neta, P., and Huie, R. E.: Rate constants for reactions of nitrate $\left(\mathrm{NO}_{3}\right)$ radicals with organic compounds in water and acetonitrile, J. Phys. Chem., 97, 3780-3782, https://doi.org/10.1021/j100117a025, 1993.

Ammann, M., Cox, R. A., Crowley, J. N., Jenkin, M. E., Mellouki, A., Rossi, M. J., Troe, J., and Wallington, T. J.: Evaluated kinetic and photochemical data for atmospheric chemistry: Vol- ume VI - heterogeneous reactions with liquid substrates, Atmos. Chem. Phys., 13, 8045-8228, https://doi.org/10.5194/acp13-8045-2013, 2013.

Arakaki, T., Saito, K., Okada, K., Nakajima, H., and Hitomi, Y.: Contribution of fulvic acid to the photochemical formation of $\mathrm{Fe}(\mathrm{II})$ in acidic Suwannee River fulvic acid solutions, Chemosphere, 78, 1023-1027, https://doi.org/10.1016/j.chemosphere.2009.11.035, 2010.

Arakaki, T., Anastasio, C., Kuroki, Y., Nakajima, H., Okada, K., Kotani, Y., Handa, D., Azechi, S., Kimura, T., Tsuhako, A., and Miyagi, Y.: A general scavenging rate constant for reaction of hydroxyl radical with organic carbon in atmospheric waters, Environ. Sci. Technol., 47, 8196-8203, https://doi.org/10.1021/es401927b, 2013.

Asmus, K. D., Möckel, H., and Henglein, A.: Pulse radiolytic study of site of $\mathrm{OH}$ radical attack on aliphatic alcohols in aqueous solution, J. Phys. Chem., 77, 1218-1221, https://doi.org/10.1021/j100629a007, 1973.

Aumont, B., Szopa, S., and Madronich, S.: Modelling the evolution of organic carbon during its gas-phase tropospheric oxidation: development of an explicit model based on a self generating approach, Atmos. Chem. Phys., 5, 2497-2517, https://doi.org/10.5194/acp-5-2497-2005, 2005.

Bean, J. K., Faxon, C. B., Leong, Y. J., Wallace, H. W., Cevik, B. K., Ortiz, S., Canagaratna, M. R., Usenko, S., Sheesley, R. J., Griffin, R. J., and Ruiz, L. H.: Composition and sources of particulate matter measured near Houston, TX: Anthropogenic-biogenic interactions, Atmosphere, 7, 1-23, https://doi.org/10.3390/atmos7050073, 2016.

Benson, S. W.: Thermochemical kinetics: methods for the estimation of thermochemical data and rate parameters, 2nd ed., edited by: Benson, S. W., John Wiley \& Sons, Inc., New York, 1976.

Bonifaciić, M., Armstrong, D. A., Štefanić, I., and Asmus, K.-D.: Kinetic isotope effect for hydrogen abstraction by $\cdot \mathrm{OH}$ radicals from normal and carbon-deuterated ethyl alcohol and methylamine in aqueous solutions, J. Phys. Chem. B, 107, 7268-7276, https://doi.org/10.1021/jp027790e, 2003.

Boucher, O., Randall, D., Artaxo, P., Bretherton, C., Feingold, G., Forster, P., Kerminen, V.-M., Kondo, Y., Liao, H., Lohmann, U., Rasch, P., Satheesh, S. K., Sherwood, S., Stevens, B., and Zhang, X. Y.: Clouds and Aerosols, in: Climate Change 2013: The Physical Science Basis, Contribution of Working Group I to the Fifth Assessment Report of the Intergovernmental Panel on Climate Change, edited by: Stocker, T. F., Qin, D., Plattner, G.-K., Tignor, M., Allen, S. K., Boschung, J., Nauels, A., Xia, Y., Bex, V., and Midgley, P. M., 571-658, Cambridge University Press, Cambridge, UK, 2013.

Brasseur, G. P., Artaxo, P., Barrie, L. A., Delmas, R. J., Galbally, I. E., Hao, W. M., Harriss, R. C., Isaksen, I. S. A., Jacob, D. J., Kolb, C. E., Prather, M., Rodhe, H., Schwela, D., Steffen, W., and Wuebbles, D. J.: Chapter 7, An Integrated View of Causes and Impacts of Atmospheric Changes, in: Atmospheric Chemistry in a Changing World - An Integration and Synthesis of a Decade of Tropospheric Chemistry Research, edited by: Brasseur, G. P., Prinn, R. G., and Pszenny, A. A. R., Springer Verlag, Berlin, Germany, 2003.

Bräuer, P., Tilgner, A., Wolke, R., and Herrmann, H.: Mechanism development and modelling of tropospheric multiphase halogen chemistry: The CAPRAM Halogen Module 2.0 (HM2), J. 
Atmos. Chem., 70, 1-34, https://doi.org/10.1007/s10874-0139249-6, 2013.

Brégonzio-Rozier, L., Giorio, C., Siekmann, F., Pangui, E., Morales, S. B., Temime-Roussel, B., Gratien, A., Michoud, V., Cazaunau, M., DeWitt, H. L., Tapparo, A., Monod, A., and Doussin, J.-F.: Secondary organic aerosol formation from isoprene photooxidation during cloud condensationevaporation cycles, Atmos. Chem. Phys., 16, 1747-1760, https://doi.org/10.5194/acp-16-1747-2016, 2016.

Buxton, G. V., Greenstock, C. L., Helman, W. P., and Ross, A. B.: Critical review of rate constants for reactions of hydrated electrons, hydrogen atoms and hydroxyl radicals $\left(\mathrm{OH} / \mathrm{O}^{-}\right)$in aqueous solution, J. Phys. Chem. Ref. Data, 17, 513-886, 1988.

Compernolle, S. and Müller, J.-F.: Henry's law constants of diacids and hydroxy polyacids: recommended values, Atmos. Chem. Phys., 14, 2699-2712, https://doi.org/10.5194/acp-142699-2014, 2014a.

Compernolle, S. and Müller, J.-F.: Henry's law constants of polyols, Atmos. Chem. Phys., 14, 12815-12837, https://doi.org/10.5194/acp-14-12815-2014, 2014b.

Davidovits, P., Kolb, C. E., Williams, L. R., Jayne, J. T., and Worsnop, D. R.: Mass Accommodation and Chemical Reactions at Gas-Liquid Interfaces, Chem. Rev., 106, 1323-1354, https://doi.org/10.1021/cr040366k, 2006.

Davidovits, P., Kolb, C. E., Williams, L. R., Jayne, J. T., and Worsnop, D. R.: Update 1 of: Mass Accommodation and Chemical Reactions at Gas-Liquid Interfaces, Chem. Rev., 111, 76-109, https://doi.org/10.1021/cr100360b, 2011.

Day, D. A., Liu, S., Russell, L. M., and Ziemann, P. J.: Organonitrate group concentrations in submicron particles with high nitrate and organic fractions in coastal southern California, Atmos. Environ., 44, 1970-1979, https://doi.org/10.1016/J.ATMOSENV.2010.02.045, 2010.

Doussin, J.-F. and Monod, A.: Structure-activity relationship for the estimation of $\mathrm{OH}$-oxidation rate constants of carbonyl compounds in the aqueous phase, Atmos. Chem. Phys., 13, 1162511641, https://doi.org/10.5194/acp-13-11625-2013, 2013.

Dunmore, R. E., Hopkins, J. R., Lidster, R. T., Lee, J. D., Evans, M. J., Rickard, A. R., Lewis, A. C., and Hamilton, J. F.: Diesel-related hydrocarbons can dominate gas phase reactive carbon in megacities, Atmos. Chem. Phys., 15, 9983-9996, https://doi.org/10.5194/acp-15-9983-2015, 2015.

Ervens, B.: Modeling the Processing of Aerosol and Trace Gases in Clouds and Fogs, Chem. Rev., 115, 4157-4198, https://doi.org/10.1021/cr5005887, 2015.

Ervens, B. and Kreidenweis, S. M.: SOA Formation by Biogenic and Carbonyl Compounds?: Data Evaluation and Application, Environ. Sci. Technol., 41, 3904-3910, https://doi.org/10.1021/es061946x, 2007.

Ervens, B., Turpin, B. J., and Weber, R. J.: Secondary organic aerosol formation in cloud droplets and aqueous particles (aqSOA): a review of laboratory, field and model studies, Atmos. Chem. Phys., 11, 11069-11102, https://doi.org/10.5194/acp-1111069-2011, 2011.

Evans, M. G.: Thermodynamical treatment of transition state, Trans. Faraday Soc., 34, 49-57, https://doi.org/10.1039/TF9383400049, 1938.
Fuller, E. N.: Diffusion coefficients for binary gas systems at low pressures: Empirical correlations, McGraw Hill, New York, 1986.

George, C., Sidebottom, H. W., Mellouki, A., Barnes, I., Pilling, M. J., Herrmann, H., Wortham, H., Kirchner, F., Wirtz, K., Zetzsch, C., and Kanakidou, M.: Final report (sections 1-6), Fifth Framework Programme 1998-2002, Thematic Programme: Environment and Sustainable Development, Project number EVK2CT-2001-00114., 2005.

Goldstein, A. H. and Galbally, I. E.: Known and unexplored organic constituents in the earth's atmosphere, Environ. Sci. Technol., 41, 1514-1521, https://doi.org/10.1021/es072476p, 2007.

Haag, W. R. and Yao, C. C. D.: Rate constants for reaction of hydroxyl radicals with several drinking water contaminants, Environ. Sci. Technol., 26, 1005-1013, https://doi.org/10.1021/es00029a021, 1992.

Hallquist, M., Wenger, J. C., Baltensperger, U., Rudich, Y., Simpson, D., Claeys, M., Dommen, J., Donahue, N. M., George, C., Goldstein, A. H., Hamilton, J. F., Herrmann, H., Hoffmann, T., Iinuma, Y., Jang, M., Jenkin, M. E., Jimenez, J. L., Kiendler-Scharr, A., Maenhaut, W., McFiggans, G., Mentel, Th. F., Monod, A., Prévôt, A. S. H., Seinfeld, J. H., Surratt, J. D., Szmigielski, R., and Wildt, J.: The formation, properties and impact of secondary organic aerosol: current and emerging issues, Atmos. Chem. Phys., 9, 5155-5236, https://doi.org/10.5194/acp9-5155-2009, 2009.

Herrmann, H.: Kinetics of Aqueous Phase Reactions Relevant for Atmospheric Chemistry, Chem. Rev., 103, 4691-4716, https://doi.org/10.1021/cr020658q, 2003.

Herrmann, H.: On the photolysis of simple anions and neutral molecules as sources of $\mathrm{O}^{-} / \mathrm{OH}, \mathrm{SO}_{x}^{-}$and $\mathrm{Cl}$ in aqueous solution, Phys. Chem. Chem. Phys., 9, 3935-3964, https://doi.org/10.1039/B618565G, 2007.

Herrmann, $\mathrm{H}$. and Zellner, R.: Reactions of $\mathrm{NO}_{3}$-radicals in aqueous solution, edited by: Alfassi, Z. B., 291-343, Wiley, New York, 1998.

Herrmann, H., Tilgner, A., Barzaghi, P., Majdik, Z., Gligorovski, S., Poulain, L., and Monod, A.: Towards a more detailed description of tropospheric aqueous phase organic chemistry: CAPRAM 3.0, Atmos. Environ., 39, 4351-4363, https://doi.org/10.1016/j.atmosenv.2005.02.016, 2005.

Herrmann, H., Hoffmann, D., Schaefer, T., Bräuer, P., and Tilgner, A.: Tropospheric Aqueous-Phase Free-Radical Chemistry: Radical Sources, Spectra, Reaction Kinetics and Prediction Tools, Chem. Phys. Chem., 11, 3796-3822, 2010.

Hoffmann, D., Weigert, B., Barzaghi, P., and Herrmann, H.: Reactivity of poly-alcohols towards $\mathrm{OH}, \mathrm{NO}_{3}$ and $\mathrm{SO}_{4}^{-}$ in aqueous solution, Phys. Chem. Chem. Phys., 11, 9351, https://doi.org/10.1039/b908459b, 2009.

Iglewicz, B. and Hoaglin, D.: Volume 16: How to Detect and Handle Outliers, The ASQC Basic References in Quality Control: Statistical Techniques, edited by: Mykytka, E. F., Ph.D., 1993.

Jenkin, M. E., Saunders, S. M., and Pilling, M. J.: The tropospheric degradation of volatile organic compounds: A protocol for mechanism development, Atmos. Environ., 31, 81-104, https://doi.org/10.1016/S1352-2310(96)00105-7, 1997.

Jenkin, M. E., Saunders, S. M., Wagner, V., and Pilling, M. J.: Protocol for the development of the Master Chemical Mechanism, MCM v3 (Part B): tropospheric degradation of aromatic 
volatile organic compounds, Atmos. Chem. Phys., 3, 181-193, https://doi.org/10.5194/acp-3-181-2003, 2003.

Jimenez, J. L., Canagaratna, M. R., Donahue, N. M., Prevot, A. S. H., Zhang, Q., Kroll, J. H., DeCarlo, P. F., Allan, J. D., Coe, H., Ng, N. L., Aiken, A. C., Docherty, K. S., Ulbrich, I. M., Grieshop, A. P., Robinson, A. L., Duplissy, J., Smith, J. D., Wilson, K. R., Lanz, V. A., Hueglin, C., Sun, Y. L., Tian, J., Laaksonen, A., Raatikainen, T., Rautiainen, J., Vaattovaara, P., Ehn, M., Kulmala, M., Tomlinson, J. M., Collins, D. R., Cubison, M. J., Dunlea, J., Huffman, J. A., Onasch, T. B., Alfarra, M. R., Williams, P. I., Bower, K., Kondo, Y., Schneider, J., Drewnick, F., Borrmann, S., Weimer, S., Demerjian, K., Salcedo, D., Cottrell, L., Griffin, R., Takami, A., Miyoshi, T., Hatakeyama, S., Shimono, A., Sun, J. Y., Zhang, Y. M., Dzepina, K., Kimmel, J. R., Sueper, D., Jayne, J. T., Herndon, S. C., Trimborn, A. M., Williams, L. R., Wood, E. C., Middlebrook, A. M., Kolb, C. E., Baltensperger, U., and Worsnop, D. R.: Evolution of Organic Aerosols in the Atmosphere, Science, 326, 1525-1529, https://doi.org/10.1126/science.1180353, 2009.

Kahnt, A., Iinuma, Y., Böge, O., Mutzel, A., and Herrmann, H.: Denuder sampling techniques for the determination of gasphase carbonyl compounds: A comparison and characterisation of in situ and ex situ derivatisation methods, J. Chromatogr. B, 879, 1402-1411, https://doi.org/10.1016/j.jchromb.2011.02.028, 2011.

Kames, J. and Schurath, U.: Henry's Law and hydrolysisrate constants for peroxyacyl nitrates (PANS) using a homogeneous gas-phase source, J. Atmos. Chem., 21, 151-164, https://doi.org/10.1007/BF00696578, 1995.

Kiendler-Scharr, A., Mensah, A. A., Friese, E., Topping, D., Nemitz, E., Prevot, A. S. H., Äijälä, M., Allan, J., Canonaco, F., Canagaratna, M., Carbone, S., Crippa, M., Dall Osto, M., Day, D. A., De Carlo, P., Di Marco, C. F., Elbern, H., Eriksson, A., Freney, E., Hao, L., Herrmann, H., Hildebrandt, L., Hillamo, R., Jimenez, J. L., Laaksonen, A., McFiggans, G., Mohr, C., O’Dowd, C., Otjes, R., Ovadnevaite, J., Pandis, S. N., Poulain, L., Schlag, P., Sellegri, K., Swietlicki, E., Tiitta, P., Vermeulen, A., Wahner, A., Worsnop, D., and Wu, H. C.: Ubiquity of organic nitrates from nighttime chemistry in the European submicron aerosol, Geophys. Res. Lett., 43, 7735-7744, https://doi.org/10.1002/2016GL069239, 2016.

La, Y. S., Camredon, M., Ziemann, P. J., Valorso, R., Matsunaga, A., Lannuque, V., Lee-Taylor, J., Hodzic, A., Madronich, S., and Aumont, B.: Impact of chamber wall loss of gaseous organic compounds on secondary organic aerosol formation: explicit modeling of SOA formation from alkane and alkene oxidation, Atmos. Chem. Phys., 16, 1417-1431, https://doi.org/10.5194/acp16-1417-2016, 2016.

Lee-Taylor, J., Hodzic, A., Madronich, S., Aumont, B., Camredon, M., and Valorso, R.: Multiday production of condensing organic aerosol mass in urban and forest outflow, Atmos. Chem. Phys., 15, 595-615, https://doi.org/10.5194/acp-15-595-2015, 2015.

Li, K. and Crittenden, J.: Computerized pathway elucidation for hydroxyl radical-induced chain reaction mechanisms in aqueous phase advanced oxidation processes, Environ. Sci. Technol., 43, 2831-2837, https://doi.org/10.1021/es802039y, 2009.

Mayer, B. and Madronich, S.: Actinic flux and photolysis in water droplets: Mie calculations and geometrical optics limit, Atmos.
Chem. Phys., 4, 2241-2250, https://doi.org/10.5194/acp-4-22412004, 2004.

McVay, R. C., Zhang, X., Aumont, B., Valorso, R., Camredon, M., La, Y. S., Wennberg, P. O., and Seinfeld, J. H.: SOA formation from the photooxidation of a-pinene: systematic exploration of the simulation of chamber data, Atmos. Chem. Phys., 16, 27852802, https://doi.org/10.5194/acp-16-2785-2016, 2016.

Minakata, D., Li, K., Westerhoff, P., and Crittenden, J.: Development of a group contribution method to predict aqueous phase hydroxyl radical (HO) reaction rate constants, Environ. Sci. Technol., 43, 6220-6227, https://doi.org/10.1021/es900956c, 2009.

Monod, A. and Doussin, J. F.: Structure-activity relationship for the estimation of $\mathrm{OH}$-oxidation rate constants of aliphatic organic compounds in the aqueous phase: alkanes, alcohols, organic acids and bases, Atmos. Environ., 42, 7611-7622, https://doi.org/10.1016/j.atmosenv.2008.06.005, 2008.

Mouchel-Vallon, C., Bräuer, P., Camredon, M., Valorso, R., Madronich, S., Herrmann, H., and Aumont, B.: Explicit modeling of volatile organic compounds partitioning in the atmospheric aqueous phase, Atmos. Chem. Phys., 13, 1023-1037, https://doi.org/10.5194/acp-13-1023-2013, 2013.

Mouchel-Vallon, C., Deguillaume, L., Monod, A., Perroux, H., Rose, C., Ghigo, G., Long, Y., Leriche, M., Aumont, B., Patryl, L., Armand, P., and Chaumerliac, N.: CLEPS 1.0: A new protocol for cloud aqueous phase oxidation of VOC mechanisms, Geosci. Model Dev., 10, 1339-1362, https://doi.org/10.5194/gmd-10-1339-2017, 2017.

Mutzel, A., Rodigast, M., Iinuma, Y., Böge, O., and Herrmann, H.: Monoterpene SOA - Contribution of firstgeneration oxidation products to formation and chemical composition, Atmos. Environ., 130, 136-144, https://doi.org/10.1016/j.atmosenv.2015.10.080, 2016.

Pereira, K. L., Dunmore, R., Whitehead, J., Alfarra, M. R., Allan, J. D., Alam, M. S., Harrison, R. M., McFiggans, G., and Hamilton, J. F.: Technical note: Use of an atmospheric simulation chamber to investigate the effect of different engine conditions on unregulated VOC-IVOC diesel exhaust emissions, Atmos. Chem. Phys., 18, 11073-11096, https://doi.org/10.5194/acp-18-110732018, 2018.

Perrin, D. D., Dempsey, B., and Serjeant, E. P.: pKa prediction for organic acids and bases, Chapman and Hall, London, UK, 1981.

Raventos-Duran, T., Camredon, M., Valorso, R., Mouchel-Vallon, C., and Aumont, B.: Structure-activity relationships to estimate the effective Henry's law constants of organics of atmospheric interest, Atmos. Chem. Phys., 10, 7643-7654, https://doi.org/10.5194/acp-10-7643-2010, 2010.

Ross, A. B., Bielski, B. H. J., Buxton, G. V, Cabelli, D. E., Helman, W. P., Huie, R. E., Grodkowski, J., Neta, P., Mulazzani, Q. G., and Wilkinson, F.: NIST Standard Reference Database 40, Version 3.0, Gaithersburg, MD, USA, 1998.

Salma, I. and Láng, G. G.: How many carboxyl groups does an average molecule of humic-like substances contain?, Atmos. Chem. Phys., 8, 5997-6002, https://doi.org/10.5194/acp-8-5997-2008, 2008.

Saunders, S. M., Jenkin, M. E., Derwent, R. G., and Pilling, M. J.: Protocol for the development of the Master Chemical Mechanism, MCM v3 (Part A): tropospheric degradation of non- 
aromatic volatile organic compounds, Atmos. Chem. Phys., 3, 161-180, https://doi.org/10.5194/acp-3-161-2003, 2003.

Schaefer, T., Schindelka, J., Hoffmann, D., and Herrmann, H.: Laboratory kinetic and mechanistic studies on the $\mathrm{OH}$-initiated oxidation of acetone in aqueous solution, J. Phys. Chem. A, 116, 6317-6326, https://doi.org/10.1021/jp2120753, 2012.

Schöne, L., Schindelka, J., Szeremeta, E., Schaefer, T., Hoffmann, D., Rudzinski, K. J., Szmigielski, R., and Herrmann, H.: Atmospheric aqueous phase radical chemistry of the isoprene oxidation products methacrolein, methyl vinyl ketone, methacrylic acid and acrylic acid - kinetics and product studies, Phys. Chem. Chem. Phys., 16, 6257-6272, https://doi.org/10.1039/C3CP54859G, 2014.

Schuchmann, H. P. and von Sonntag, C.: Methylperoxyl Radicals: A Study of the $\gamma$-Radiolysis of Methane in Oxygenated Aqueous Solutions, Zeitschrift fur Naturforsch. - Sect. B J. Chem. Sci., 39, 217-221, https://doi.org/10.1515/znb-1984-0217, 1984.

Schuchmann, M. N., Zegota, H., and von Sonntag, C.: Acetate peroxyl radicals, $\mathrm{O}_{2} \mathrm{CH}_{2} \mathrm{CO}_{2}-$ - A study on the gamma radiolysis and pulse-radiolysis of acetate in oxygenated aqueous solutions, Zeitschrift für Naturforsch. Sect. B - A J. Chem. Sci., 40, 215 221, 1985.

Schwartz, S. E.: Mass transport considerations pertinent to aqueous phase reactions of gases in liquid water clouds, edited by: Jaeschke, W., 415-471, Springer, Berlin, Germany, 1986.

Sierau, B., Stratmann, F., Pelzing, M., Neusüß, C., Hofmann, D., and Wilck, M.: A condensation-growth and impaction method for rapid off-line chemical-characterization of organic submicrometer atmospheric aerosol particles, J. Aerosol Sci., 34, 225242, https://doi.org/10.1016/S0021-8502(02)00159-3, 2003.

Tilgner, A. and Herrmann, H.: Radical-driven carbonyl-to-acid conversion and acid degradation in tropospheric aqueous systems studied by CAPRAM, Atmos. Environ., 44, 5415-5422, 2010.
Tilgner, A., Bräuer, P., Wolke, R., and Herrmann, H.: Modelling multiphase chemistry in deliquescent aerosols and clouds using CAPRAM3.0i, J. Atmos. Chem., 70, 221-256, https://doi.org/10.1007/s10874-013-9267-4, 2013.

Vereecken, L., Aumont, B., Barnes, I., Bozzelli, J. W., Goldman, M. J., Green, W. H., Madronich, S., Mcgillen, M. R., Mellouki, A., Orlando, J. J., Picquet-Varrault, B., Rickard, A. R., Stockwell, W. R., Wallington, T. J., and Carter, W. P. L.: Perspective on Mechanism Development and Structure-Activity Relationships for GasPhase Atmospheric Chemistry, Int. J. Chem. Kinet., 50, 435-469, https://doi.org/10.1002/kin.21172, 2018.

von Sonntag, C.: The chemical basis of radiation biology, Taylor and Francis, London, Great Britain, 1987.

von Sonntag, C. and Schuchmann, H. P.: The elucidation of peroxyl radical reactions in aqueous solution with the help of radiation chemical methods, Angew. Chemie - Int. Ed. English, 30, 1229 1253, https://doi.org/10.1002/anie.199112291, 1991.

von Sonntag, C., Dowideit, P., Fang, X. W., Mertens, R., Pan, X. M., Schuchmann, M. N., and Schuchmann, H. P.: The fate of peroxyl radicals in aqueous solution, Water Sci. Technol., 35, 915, https://doi.org/10.1016/S0273-1223(97)00003-6, 1997.

Wang, C., Yuan, T., Wood, S. A., Goss, K.-U., Li, J., Ying, Q., and Wania, F.: Uncertain Henry's law constants compromise equilibrium partitioning calculations of atmospheric oxidation products, Atmos. Chem. Phys., 17, 7529-7540, https://doi.org/10.5194/acp-17-7529-2017, 2017.

Wolke, R., Sehili, A. M., Simmel, M., Knoth, O., Tilgner, A., and Herrmann, H.: SPACCIM: A parcel model with detailed microphysics and complex multiphase chemistry, Atmos. Environ., 39, 4375-4388, https://doi.org/10.1016/j.atmosenv.2005.02.038, 2005. 